\title{
Lithographic band structure engineering of graphene
}

Jessen, Bjarke S.; Gammelgaard, Lene; Thomsen, Morten R.; Mackenzie, David M. A.; Thomsen, Joachim D.; Caridad, José M.; Duegaard, Emil; Watanabe, Kenji; Taniguchi, Takashi; Booth, Timothy J. Total number of authors:

13

Published in:

Nature Nanotechnology

Link to article, DOI:

$10.1038 / \mathrm{s} 41565-019-0376-3$

Publication date:

2019

Document Version

Early version, also known as pre-print

Link back to DTU Orbit

Citation (APA):

Jessen, B. S., Gammelgaard, L., Thomsen, M. R., Mackenzie, D. M. A., Thomsen, J. D., Caridad, J. M., Duegaard, E., Watanabe, K., Taniguchi, T., Booth, T. J., Pedersen, T. G., Jauho, A-P., \& Bøggild, P. (2019). Lithographic band structure engineering of graphene. Nature Nanotechnology, 14, 340-346.

https://doi.org/10.1038/s41565-019-0376-3

\section{General rights}

Copyright and moral rights for the publications made accessible in the public portal are retained by the authors and/or other copyright owners and it is a condition of accessing publications that users recognise and abide by the legal requirements associated with these rights.

- Users may download and print one copy of any publication from the public portal for the purpose of private study or research.

- You may not further distribute the material or use it for any profit-making activity or commercial gain

- You may freely distribute the URL identifying the publication in the public portal 


\title{
Lithographic band structure engineering of graphene
}

\author{
Bjarke S. Jessen, ${ }^{1,2}, *$ Lene Gammelgaard, ${ }^{1,2}, *$ Morten R. Thomsen,${ }^{3,4}$ David M. A. Mackenzie, ${ }^{1,2}$ \\ Joachim D. Thomsen, ${ }^{1,2}$ José M. Caridad, ${ }^{1,2}$ Emil Duegaard, ${ }^{1,2}$ Kenji Watanabe, ${ }^{5}$ Takashi Taniguchi, ${ }^{5}$ \\ Timothy J. Booth, ${ }^{1,2}$ Thomas G. Pedersen, ${ }^{3,4}$ Antti-Pekka Jauho, ${ }^{1,2}$ and Peter Bøggild ${ }^{1,2, \dagger}$ \\ ${ }^{1}$ Center for Nanostructured Graphene, Technical University of Denmark, DK-2800, Denmark \\ ${ }^{2}$ DTU Nanotech, Technical University of Denmark, DK-2800, Denmark \\ ${ }^{3}$ Center for Nanostructured Graphene, Aalborg University, DK-9220, Denmark \\ ${ }^{4}$ Department of Physics and Nanotechnology, Aalborg University, DK-9220, Denmark \\ ${ }^{5}$ National Institute for Materials Science, 1-1 Namiki, Tsukuba, 305-0044, Japan
}

\begin{abstract}
Nanolithography, a cornerstone of modern information technology, allows manipulation of the electronic band structure and quantum transport properties of materials. By miniaturisation and careful shaping, any material can be pushed into a (quantum) regime, where the dimensions and shape become essential for the overall behaviour, and the electronic band structure is significantly altered. Materials with a low intrinsic dimensionality, such as the two-dimensional material graphene, allow direct access to the entirety of atoms constituting the crystal, making engineering of the band structure particularly attractive. While high-density nanostructuring has been predicted to provide customisation of the electronic, optical, plasmonic, and thermal properties of graphene [1-5], there has been limited progress in realising this in practice; even high-end top-down fabrication procedures introduce enough edge-disorder and contamination to obscure the predicted behaviours. Here we demonstrate band structure engineering by direct, ultra-dense lithographic patterning of graphene. Specifically, we fabricate a $35 \mathrm{~nm}$-period superlattice of etched holes separated by as little as 12 $15 \mathrm{~nm}$ in a graphene sheet encapsulated in hexagonal boron nitride. We observe the clear hallmark of a distinct magnetotransport regime, with a nonlinear Landau level fan, and a band gap of $156 \mathrm{meV}$, which can be tuned with an external magnetic field. The transport measurements are in excellent agreement with both tight-binding simulations and an analytical model based on Dirac fermions in ring geometries [6]. A moiré superlattice from the underlying substrate is observed both before and after nanostructuring, and we see transport features unique to our engineered band structure both at the main and moiré charge neutrality points, indicating that the engineered band structure is cloned by the moiré superlattice. Band structure design in two-dimensional materials by top-down patterning enables the realisation of a number of exciting predictions and opportunities such as spin qubits [1], valleytronics [6], and waveguides [7].
\end{abstract}

Graphene is a particularly attractive material for band structure engineering, due to its bulk being directly exposed for manipulation. The three major approaches for graphene band structure engineering are $(i)$ moiré superlattices [8-10], which gives high quality results, but lacks the design flexibility of top-down lithography, (ii) indirect structuring, where the immediate surroundings are subject to nanostructuring $[11,12]$, and finally (iii) nanostructuring, which by quantum confinement effects offers the most permanent and pervasive changes of the electronic properties, as well as being essential for miniaturisation of graphene devices. To observe the full extent of these effects in electronic transport experiments, charge transport should be ballistic, with the mean free path, $l_{e}$, being larger than the minimum feature size of the system, $w$. Furthermore, the energy scale of the quantum confinement should be larger than the energy scale associated with the disorder of the system [13]. While the mean free path of pristine graphene can be tens of micrometres at cryogenic temperatures [14, 15], residual disorder limits the available system sizes. In

\footnotetext{
* These authors contributed equally to this work.

$\dagger$ E-mail:pbog@dtu.dk
}

particular, even state-of-the-art ultra-clean graphene devices show residual carrier densities in the range of $\sim$ $10^{10} \mathrm{~cm}^{-2}[16,17]$. This sets the disorder energy scale at $\hbar v_{F} \sqrt{\pi n} \sim 10 \mathrm{meV}$, which in turn, using the typical scaling of graphene nanoribbons [18], means that the minimum feature size of the system should be kept below $100 \mathrm{~nm}$ to observe the band structure changes from quantum confinement. For state-of-the-art nanostructured graphene, the residual charge carrier densities are even higher, which further restricts the required system sizes $[19,20]$.

Top-down patterning of graphene on this scale, however, is very challenging without compromising the quasiballistic condition, $l_{e} \geq w$, as has been shown for graphene nanoribbons $[13,21]$. This has led to more than a decade of research into ways of archiving dense nanostructuring of graphene without compromising the transport properties, but so far disorder at edges increasingly dominate transport measurements as pattern densities increase [22-24]. Figure 1 summarise a subset of key experimental works [19, 20, 25-31] featuring twodimensional (2D) nanostructured graphene as well as microscale Hall bars for reference [31], with the reported charge carrier mobility, $\mu$, plotted against the minimum feature size of the system. Here the mobility is calculated from the Drude model of conductivity, $\sigma=n e \mu$, 
where $e$ is the electron charge. In early devices fabricated from non-encapsulated graphene, extremely small and dense features have indeed been achieved with block copolymer (BCP) [25] masks, electron-beam lithography (EBL) [26, 29, 30], nanosphere lithography (NSL) [28], and nano-imprint lithography (NIL) [27]. However, the resulting charge carrier mobilities and associated mean free paths in these works were too low to support quantum transport of the charge carriers beyond semiclassical transport. A clear trend is seen, where higher pattern densities leads to strongly decreasing mobilities, which can be attributed to the increasing contribution of scattering from rough edges. Recently, however, progress was made using van der Waals (vdW) heterostructures of graphene encapsulated in hexagonal boron nitride (hBN) $[19,20,32]$. Patterning through vdW heterostructures not only provides protection of the graphene channel, but also the edges [19], which obviously become increasingly important at higher pattern densities. While these nanostructured heterostructures displayed clear commensurability oscillations [19, 20, 33, 34], vdW heterostructures with stronger confinement (higher pattern density) are needed to reach the quantum regime, as apparent from Fig. 1.

Here we show that band structure engineering by top-down lithographic patterning of $\mathrm{hBN}$ encapsulated graphene can be done on a $10 \mathrm{~nm}$-scale, as seen in the inset of Fig. 1, while maintaining ballistic transport properties on the scale of the minimum feature size. The patterned graphene devices display insulating behaviour consistent with the formation of an electronic band gap, and ballistic transport at Fermi energies larger than the gap. Furthermore, magnetotransport measurements match extraordinarily well with tight-binding simulations of antidot lattices as well with a simple model of Dirac fermions in a strongly confining ring geometry [6], and is, thus, well-described as a quantum system consisting of such connected "Dirac rings". Our measurements confirm several theoretical predictions for such band structure engineered graphene, including a sizeable and magnetically tunable band gap $[1,6,35]$, magnetically confined edge-states [36], and non-linear Landau levels [6]. Furthermore, despite the $10 \mathrm{~nm}$-scale patterning of the graphene, the experimental signatures of an interfacial moiré superlattice turn out to be present both before and after nanostructuring. For the nanostructured graphene, it appears that the unique transport features are cloned by the moiré superlattice, suggesting an intricate interplay of the engineered and interfacial superlattices with the electronic band structure.

The vdW heterostructures used in this study consist of monolayer graphene encapsulated in hBN on a graphite back gate. The heterostructures are shaped into multiterminal Hall bars with one half nanostructured into an array of holes, as illustrated in Fig. 2A. Full details of the fabrication and nanostructuring can be found in the Methods and Supplementary Information. This geometry allows us to simultaneously measure the longitudinal

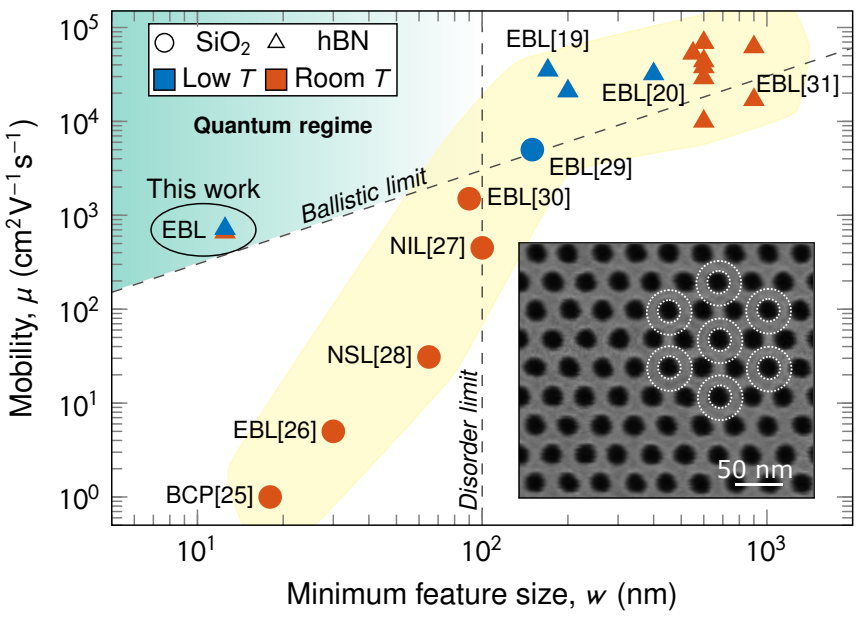

Figure 1. Charge carrier mobility, $\mu$, versus minimum feature size, $w$, for nanostructured graphene reported in the literature: For a device to be fully in the quantum regime (green shaded area), it should display ballistic transport (horizontally dashed line) at energy scales larger than the disorder limit (vertically dashed line). As the energy scale associated with quantum confinement is inversely proportional to the minimum feature size, aggressive scaling is the most feasible route towards the quantum regime. However, as conventional devices are scaled down, edge roughness starts to dominate the transport properties, pushing them away from the ballistic limit. The inset shows a SEM image of the van der Waals heterostructure used in this study after nanostructuring has been performed. The pattern consists of highly regular, triangularly arranged holes with a period of $35 \mathrm{~nm}$ and minimum feature sizes of $w=12-15 \mathrm{~nm}$. Due to the high density of holes, the resulting structure can be considered as a network of connected rings of width $w$, as indicated by the dotted outlines. These structures display ballistic transport despite the extreme density of edges, indicating a very low line-edge roughness.

and transverse resistances, $R_{x x}$ and $R_{x y}$, of the pristine and nanostructured graphene in a single device, thus directly measuring the effect of the nanostructuring. Figure $2 \mathrm{~B}$ shows the temperature dependence of the longitudinal resistivity, $\rho_{x x}=(W / L) R_{x x}$, against back gate voltage, $V_{G}$, of pristine and nanostructured graphene, with $L=4 \mu \mathrm{m}$ and $W=2 \mu \mathrm{m}$ being the distance between voltage probes and width of the channel, respectively. After nanostructuring the graphene is slightly pdoped relative to the pristine graphene, an effect which has previously been reported in nanostructured graphene [37]. The pristine graphene shows a small increase in resistivity near the Dirac point upon cooling down to $4 \mathrm{~K}$, but is otherwise metallic, as expected. In contrast, the nanostructured part of the device exhibits strongly insulating behaviour in a wide range of gate voltages around the Dirac point, with a significant increase in resistance upon cooling. This insulating behaviour is also observed in the magnitude of the resistivity, $\rho_{x x}>h / e^{2}$, which is consistent with the Ioffe-Regel criterion for $2 \mathrm{D}$ semiconductors [38, 39], where $h$ and $e$ are Planck's constant 


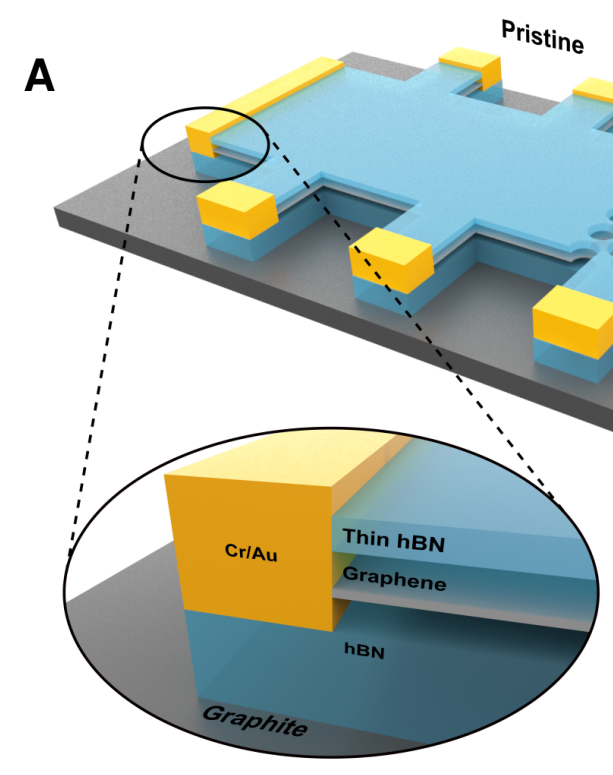

B
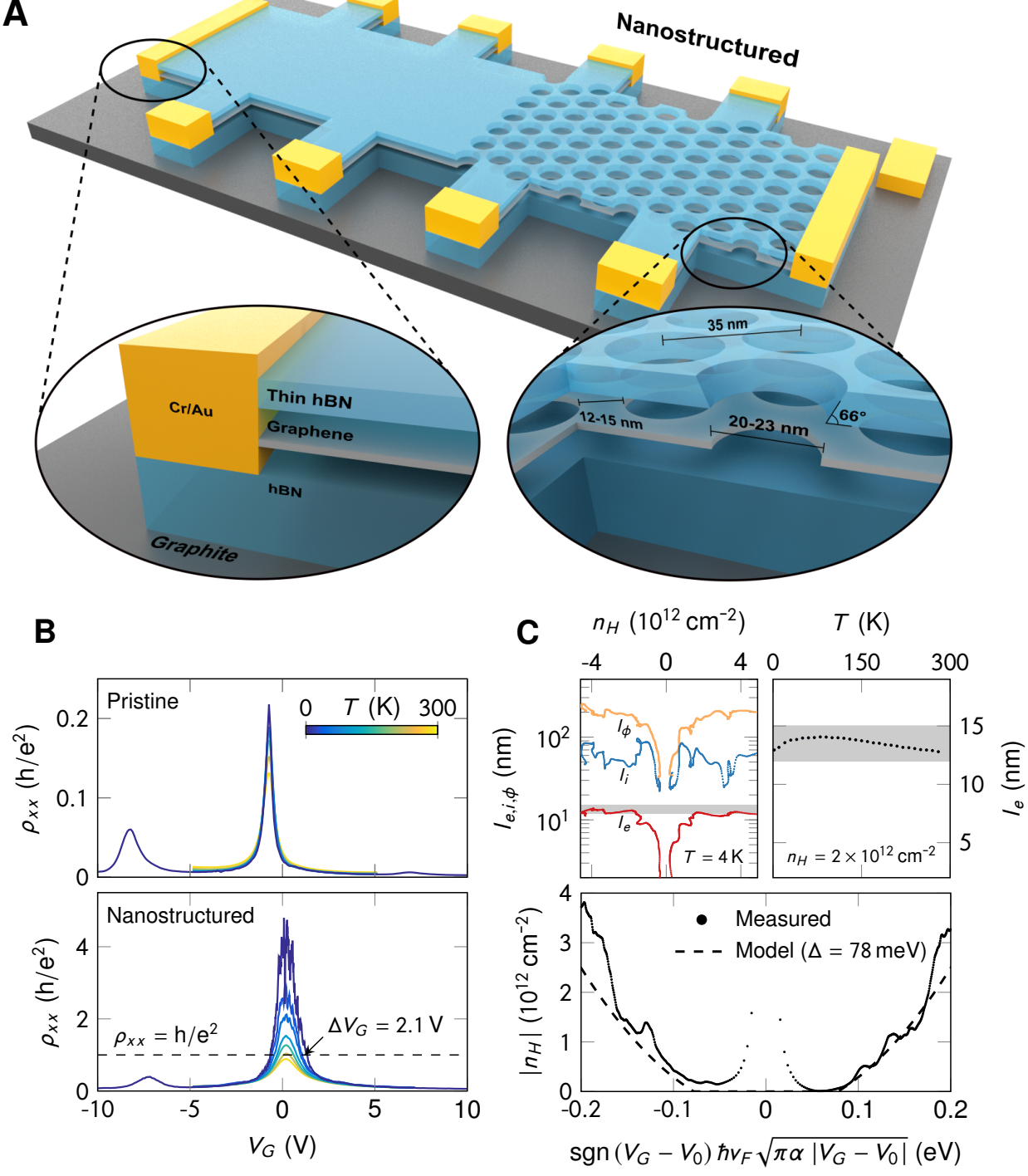

Figure 2. Device architecture and transport data for pristine and nanostructured graphene: (A) Device schematic of the Hall bar used for electrical measurements. Part of the heterostructure is left pristine, while another part is shaped into a densely patterned superlattice, allowing simultaneous comparison of patterned and non-patterned regions in a single device. (B) Resistivity, $\rho_{x x}$, against back gate voltage, $V_{G}$, for temperatures, $T$, from $4 \mathrm{~K}$ to $300 \mathrm{~K}$ for the pristine and nanostructured graphene. The patterned region exhibits an insulating regime with $\Delta V_{G} \approx 2.1 \mathrm{~V}$. (C) The upper left panel shows the extracted mean free path, $l_{e}$, intervalley-scattering length, $l_{i}$, and phase-coherence length, $l_{\phi}$, of the nanostructured region, plotted against the measured Hall charge carrier density, $n_{H}$, at $T=4 \mathrm{~K}$, while the right panel shows $l_{e}$ against $T$ at $n_{H}=2 \times 10^{12} \mathrm{~cm}^{-2}$. The shaded areas mark the $12-15 \mathrm{~nm}$ range, corresponding to the minimum feature size of the nanostructured area, indicative of ballistic transport. The lower panel shows $n_{H}$ compared to the calculated carrier density for the gapped graphene model, where $\alpha=5.87 \times 10^{11} \mathrm{~cm}^{-2} \mathrm{~V}^{-1}$ is the lever constant and $V_{0}$ is the gate voltage at the charge neutrality point.

and the electron charge, respectively. The width of the insulating regime, $\Delta V_{G} \approx 2.1 \mathrm{~V}$, can be used to estimate $[21,40]$ the energy gap, $E_{G}=2 \Delta_{0}$, in the nanostructured graphene (Supplementary Information), yielding $\Delta_{0} \approx 78 \mathrm{meV}$. Here the half-gap, $\Delta_{0}$, is the energy from the Fermi level to either the top of the conduction band or the bottom of the valence band. From Arrhenius measurements we extract a small moiré-induced half-gap $\left(\Delta_{m} \approx 14 \pm 1 \mathrm{meV}\right)$ in both the pristine and nanostruc- tured regions. In addition to this, the high temperature data for the nanostructured graphene corresponds to a half-gap of $74 \pm 11 \mathrm{meV}$ (Supplementary Information). Considering the unusually large charge carrier mobility for such small and dense nanostructuring, as shown in Fig. 1, we equate the insulating behaviour with the formation of a band gap in the electronic spectrum, as theoretically predicted for such systems $[1,6,36]$. Gapped behaviour is also observed in the measured Hall charge 
carrier density, as seen in the lower panel of Fig. 2C. The measured density fits well with the calculated charge carrier density for explicitly gapped graphene, with a halfgap of $78 \mathrm{meV}$ (Supplementary Information). As we show later, this gap also matches well with the band gap extracted from an analytical model of Dirac fermions confined to a ring of width $w$, where the half-gap is given by $\Delta_{0}=1 \mathrm{eVnm} / w$. With an estimated $w=12-15 \mathrm{~nm}$ of our structures, the analytical model predicts a halfgap of $67-83 \mathrm{meV}$. We further note that we observe a large $\rho_{x y}$ signal of $\sim 0.3 h / e^{2}$ at $B=0 \mathrm{~T}$, as shown in the Supplementary Information. Such a signal is indeed expected from a gap-induced non-zero Berry-curvature [41], but further experiments are needed to fully confirm this. Regarding the ballistic behaviour, the mean free path of charge carriers, $l_{e}$, in the nanostructured graphene is nearly constant at all measured temperatures and charge carrier densities away from the insulating region. As shown in Fig. $2 \mathrm{C}, l_{e}$ is clearly reaching the minimum feature size of out system, and indicates ballistic transport and low amounts of disorder, despite the dense nanostructuring. Here we have used $l_{e}=\sigma h / 2 e^{2} k_{F}$, where $k_{F}=\sqrt{\pi n_{H}}$ is the Fermi wavevector, $\sigma$ is the conductivity, and $n_{H}$ is the measured Hall charge carrier density from $R_{x y}=-B / n_{H} q$, with the charge, $q$, and non-quantising magnetic field $B$. We attribute the high quality of our transport to extremely low edge roughness, as indicated by the high intervalley scattering length, $l_{i}$, on the order of $50-60 \mathrm{~nm}$, which is significantly higher than any relevant length scale of our system. Finally, for the pristine graphene we observe satellite peaks in the resistance at gate voltages of $\sim \pm 7.6 \mathrm{~V}$ relative to the main charge neutrality point, consistent with a the presence of a moiré lattice induced by a slight misalignment between the graphene and the encapsulating hBN. Surprisingly, for the nanostructured graphene, the satellite peak on the hole side is still present despite the dense nanostructuring.

Next, we perform magnetotransport measurements, measuring the longitudinal conductivity, $\sigma_{x x}=$ $\rho_{x x} /\left(\rho_{x x}^{2}+\rho_{x y}^{2}\right)$, as a function of $V_{G}$ and the applied magnetic field, $B$. The resulting $\sigma_{x x}$ of both the pristine and nanostructured graphene is shown in Fig. 3. The pristine graphene displays the expected linear Landau level sequence of monolayer graphene, along with the now routinely reported moiré superlattice effects consistent with a small twist angle with respect to the hBN [8-10]. The overall shape and magnitude of the magnetotransport data in Fig. 3A, including the characteristic asymmetry on the hole and electron side, is closely resembling the data presented in Ref. [9]. We observe additional satellite peaks corresponding to moiré periods of $\lambda_{m, 1}=10.2 \mathrm{~nm}$, and of $\lambda_{m, 2}=17.2 \mathrm{~nm} \approx \sqrt{3} \lambda_{m, 1}$. The possible origins of periods larger than the oftenquoted $14 \mathrm{~nm}$ limit [42] are discuss in the Supplementary Information. The nanostructured graphene, on the other hand, exhibits energy levels, for which carrier density varies non-linearly with the magnetic field, and which intersect the zero-field line at non-zero gate values (indicating $E \neq 0$ ). Furthermore, the band-gap-induced insulating regime discussed above and in Fig. 2 is seen to be tunable by the magnetic field, and is decreased as the magnetic field strength is increased. Finally, a splitting of the zero-energy level independent of the magnetic field is observed, which is sometimes seen in high-quality samples with a small moiré gap $[10,16,17]$. Despite the famously robust quantum Hall effect in graphene $[43,44]$, all of the prominent features of the nanostructured graphene clearly deviate from the pristine graphene Landau fan. Interestingly, despite the ultra-dense nanostructuring, the moiré effects corresponding to the $10.2 \mathrm{~nm}$ period are still present, while the $17.2 \mathrm{~nm}$ effects are missing. We speculate that the effects of the $17.2 \mathrm{~nm} \mathrm{sec-}$ ondary moiré period are quenched since they exceed the minimum feature size of the nanostructured graphene. However, the exact cause warrants further investigation, and we are not aware of any theoretical investigations into this interplay.

To gain insight into the observed quantum transport behaviour of the nanostructured graphene, we compare our data to ensemble-averaged recursive Green's function tight-binding transport simulations [45, 46] in Fig. 4A, as outlined in Methods. Without any fitting, the simulations match extraordinarily well with the measurements, both in terms of shape, position, and magnitude of the observed bands. We take this agreement as evidence that the calculated band structure of the quantum simulations accurately reflects the engineered band structure of the nanostructured graphene. Furthermore, we observe socalled magnetically bound states, confined to the perimeter of the nanostructured holes. Semiclassically, these states correspond to periodic skipping orbits, as shown schematically in Fig. 4B, and are analogous to Weiss oscillations [47]. In experiments, certain conditions must be met for these states to be present: $(i)$ a skipping orbit with an integer number of reflections, $n_{r}$, along the perimeter of the hole, $(i i)$ the Fermi level is not located

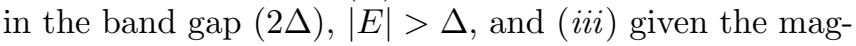
netic field, the wave function must be in phase after one orbit. Given these conditions, the oscillation period of the magnetoresistance can be shown to follow [36]

$$
\Delta B=\frac{8 \hbar}{e\left(2 \pi R_{1}\right)^{2}},
$$

where $R_{1}$ is the radius of the hole. Figure $4 \mathrm{C}$ shows part of $\rho_{x} x$ from the nanostructured graphene, where we observe oscillations with a spacing of $\Delta B \approx 1.2 \mathrm{~T}$. Using Eq. (1) this in turn corresponds to a diameter of $\sim 21 \mathrm{~nm}$, matching well our expected feature sizes.

The agreement between measurements and tightbinding simulations prompts us to further compare the data with a simplified analytical model that describes Dirac fermions confined to a ring geometry, i.e. a Dirac ring. This allows us to establish a simple physical picture of the observed magnetotransport features. The resulting eigenvalue spectrum as a function of energy and 

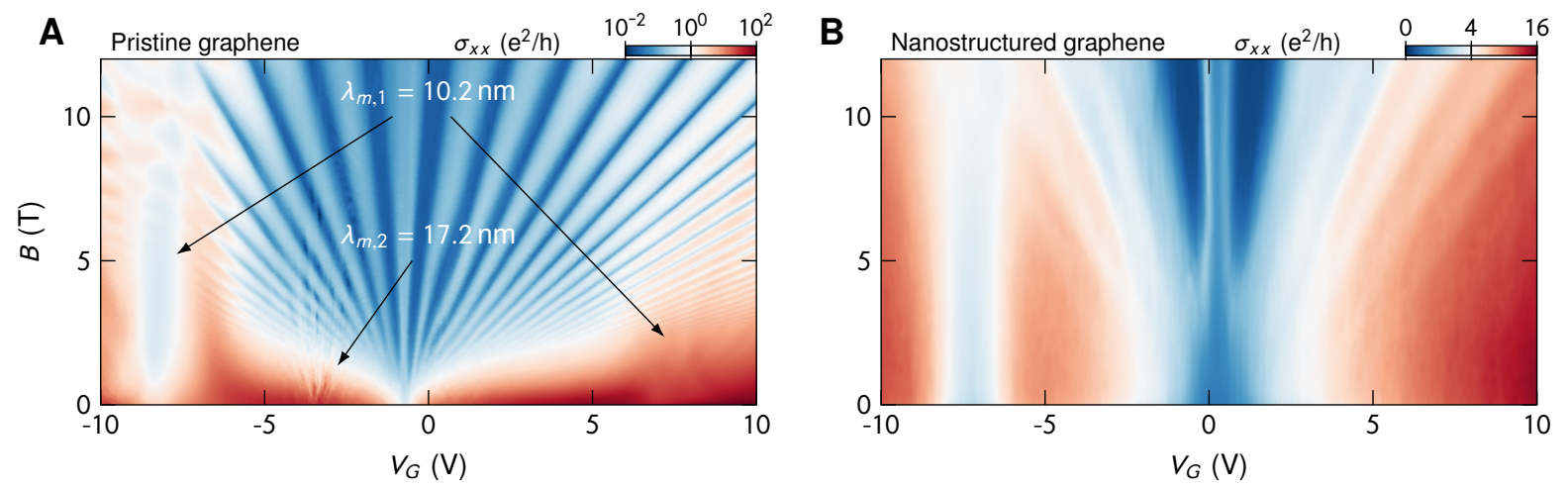

Figure 3. Comparison of magnetotransport in pristine and nanostructured graphene: Comparison of longitudinal conductivity, $\sigma_{x x}$, in the pristine $(\mathbf{A})$ and nanostructured $(\mathbf{B})$ regions of the graphene. In contrast to the linear energy levels characteristic of pristine graphene, the energy levels in the patterned region are non-linear and intersect the $B=0 \mathrm{~T}$ axis at $E \neq 0$. Furthermore, a tunable band gap (low $\sigma_{x x}$ ) is seen in the nanostructured graphene, whose magnitude decreases as the magnetic field strength is increased.

magnetic field can be seen in Fig. 4D, for a ring with an inner and outer radius of $11 \mathrm{~nm}$ and $23 \mathrm{~nm}$, respectively. The red (blue) bands corresponding to $K\left(K^{\prime}\right)$ valley-polarised states, and each band corresponds to a unique set of a radial and momentum quantum number, $n$ and $m$, respectively. Details of the model are given in the Supplementary Information. The model captures all the main features of the measurements, including energy levels which varies non-linearly with the magnetic field, energy levels that intersect at $E \neq 0$, and the presence of a band gap which is tunable by the strength of the applied magnetic field. Specifically, the model predicts a zero-field half-gap at $B=0 \mathrm{~T}$ given solely by the width of the Dirac ring, of $\Delta_{0}=1 \mathrm{eVnm} / w$. The sub-linear bands intersect at $E \neq 0$, in contrast to the linear Landau levels of pristine graphene, in agreement with our measurements, see Fig. 4A. For the Dirac ring, emergent bands intersect at $E=l \Delta_{0}$, with $l$ being an odd integer, and with $\Delta_{0}$, thus, serving as the characteristic energyscale. While the $K$ and $K^{\prime}$ valleys are degenerate at zero magnetic fields, the degeneracy is lifted at finite magnetic fields, with the top of the $K$-valley valence band increasing in energy, while the bottom of the $K^{\prime}$-valley conduction band decreases in energy as the magnetic field strength is increased. This shows that the Dirac rings could be an interesting system for valley-electronics.

In Fig. 4E we directly compare the position of the observed bands from experiments, tight-binding, and the analytical model, and find a striking agreement, especially at lower values of $V_{G}$. For the analytical model, we do not observe all the available energy levels, but do find a strong agreement for the quantum numbers $n=1$ and $0 \leq m \leq 6$. For the Dirac ring model, the $m<0$ quantum numbers corresponds to energy levels predominantly localised at the outer perimeter [6], which can explain why these states are not observed in the experiments which are not isolated rings.

The tunability of the band gap is shown in Fig. 4F, where we directly compare the half-gaps from the exper- iments, tight-binding, and the analytical model. Here the half-gap is normalised to the zero-field half-gap as $\Delta / \Delta_{0}$, and plotted against the ratio of the minimum feature size to the magnetic length, $l_{B}=\sqrt{h / e B}$. With this scaling any ring of the analytical model, regardless of inner and outer radius, will trace the same line, and vice versa for the tight-binding model. We again observe a strong agreement between the tight-binding and the experiments, while the band gap predicted by the analytical model closes much slower. As the ratio $w / l_{B}$ increases, the charge carriers are increasingly localised by the magnetic field, to the point where they no longer feel the effect of the confinement, which in turn closes the band gap. The behaviour of the gap closing in the analytical model is given by the $m<0$ energy levels which, cf. the previous discussion, are not observed in the experiments, and could account for the discrepancy. Tunable band gaps are expected to vanish in the presence of disorder $[35,36]$, further indicating that the structures considered in our work have extraordinarily low edge-roughness.

Finally, we turn our attention to the possibly most striking feature of our data, which is the presence of the moiré-induced satellite peak present after nanostructuring. In the pristine graphene, the influence of the moiré-induced potential causes second-generation Dirac cones to appear near the supercell Brillouin zone edges [48], which subsequently forms their own Landau levels under the application of a magnetic field. However, in our nanostructured graphene, the behaviour at the satellite peak is markedly different - instead of secondgeneration massless Dirac fermions, the behaviour is similar to that of the main neutrality point of the nanostructured graphene, see Fig. 5. In particular, the magnitude and width (in $V_{G}$ ) of the insulating region decreases upon increasing magnetic field. Here it is clear that the magnetoresistance of the two peaks in the pristine graphene is positive, as expected for graphene, while the nanostructured graphene exhibits a clear negative magnetore- 

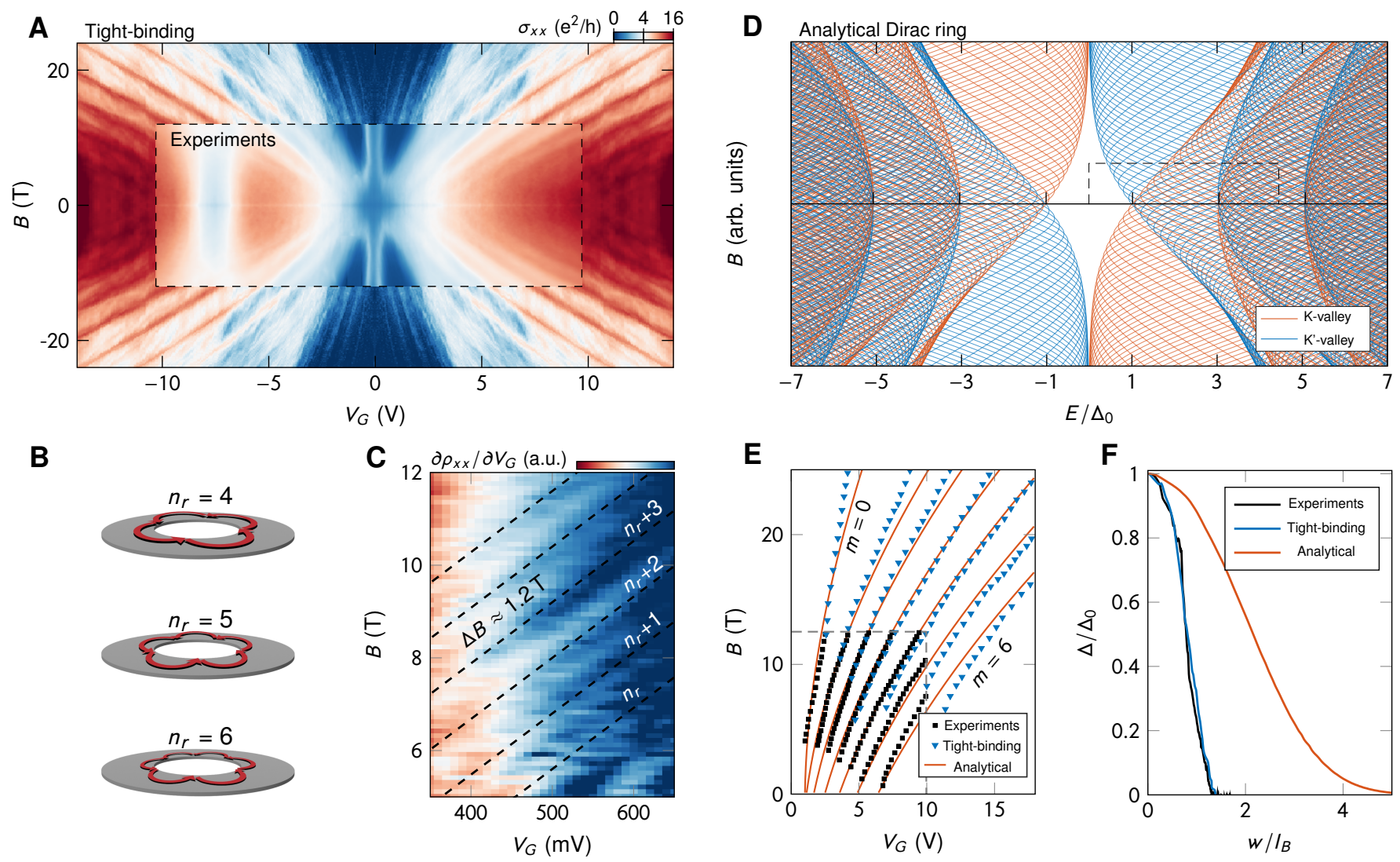

Figure 4. Magnetotransport and band gap tuning: (A) Direct comparison of $\sigma_{x x}$ from tight-binding transport simulations and experiments, showing quantitative agreement of both magnitude and position of the energy levels. (B,C) The perimeter of high-quality graphene nanostructures supports periodic, discrete energy levels corresponding to electronic states confined by a magnetic field. Semi-classically, these orbits corresponds to phase-coherent skipping orbits around the perimeter of a hole. In magnetotransport measurements, these orbits manifests themselves as periodic oscillations. From semi-classical analysis the spacing of $\approx 1.2 \mathrm{~T}$ (dashed lines of $\mathbf{C}$ ) corresponds to a diameter of $21 \mathrm{~nm}$, matching the estimated diameter of the holes in our nanostructured graphene. (D) Analytical energy levels for a Dirac ring with an inner hole diameter of $22 \mathrm{~nm}$ and a width of $12 \mathrm{~nm}$. The $B=0 \mathrm{~T}$ half-gap, $\Delta_{0}=1 \mathrm{eVnm} / w$, serves as the characteristic energy scale for all such Dirac rings, where $w$ is the width of the ring. In particular, all the emergent bands, consisting of many individual energy levels, are approximately spaced by $2 \Delta_{0}$ at $B=0 \mathrm{~T}$. Red (blue) bands correspond to $K\left(K^{\prime}\right)$ valley-polarised states. As in the experiments, the Dirac rings also feature a band gap which decreases in magnitude as the magnetic field strength is increased. (E) Direct comparison between the energy levels observed in the experiments, tight-binding transport simulations, and the analytical model. The range of gate voltages and magnetic field strength used corresponds to the dashed region in (D). Not all eigenvalues of the analytical model are observed, but eigenvalues of radial quantum number $n=1$ and angular-momentum quantum number $0 \leq m \leq 6$ are seen to agree well with experiments and simulations. (F) Direct comparison of the tunable band gap for the experiments, tight-binding simulations, and analytical model. The gap is normalised to the zero-field gap, and plotted against the ratio of the minimum feature size to the magnetic length, $l_{B}$. In this plot, any solution to the analytical model will trace the same line, and vice versa for the tight-binding simulations. Experiments and simulations are seen to agree remarkably well, while the band gap of the analytical model closes more slowly with increasing magnetic field.

sistance in both peaks, associated with the closing band gap. From tight-binding calculations it has been shown that the details of the charge carriers at the main neutrality point, such as Fermi velocity or sublattice symmetry breaking, directly influences the higher energy secondgeneration Dirac fermions [49, 50]. Furthermore, from magnetic focusing experiments it has been shown that the specific sample geometry also influences the secondgeneration Dirac fermions [51]. Since the behaviour at the main neutrality point in our sample is also governed by the sample geometry, it stands to reason that this should also govern the second-generation Dirac fermions. While it is generally believed [9] that moiré superlattices lead to self-similar transport behaviour, at this point in time, however, it is unclear to which degree the substrateinduced moiré-interaction is expected to clone the features of the main neutrality point in systems with substantially engineered band structures such as ours.

The highly regular, low edge-disorder quality of our nanostructured graphene, along with the excellent agreement with quantum simulations and analytical models clearly demonstrates that top-down fabrication is a vi- 


\section{Pristine}

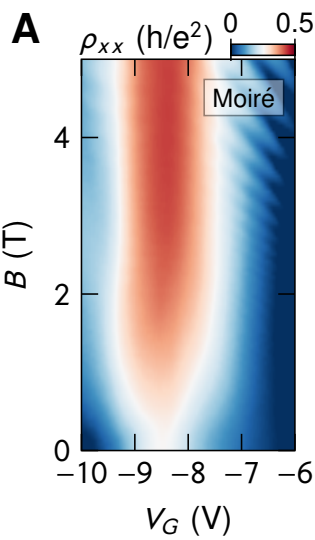

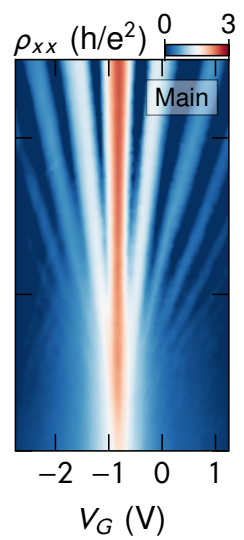

Nanostructured

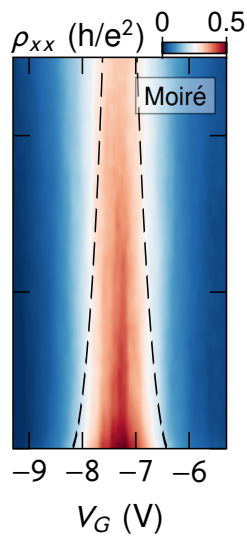

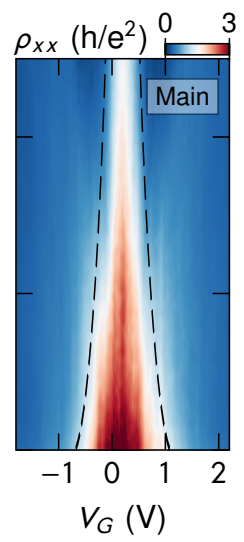

B

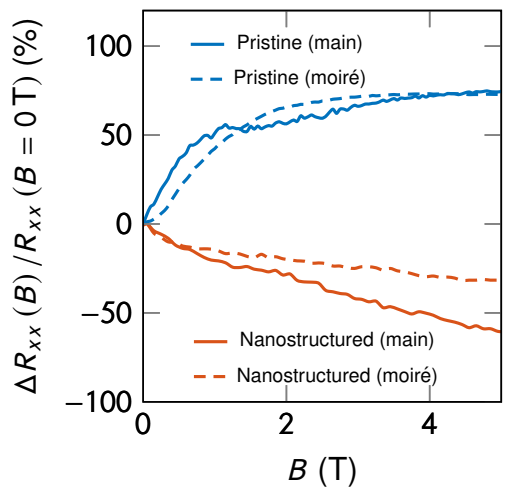

Figure 5. Cloning of engineered band structure: (A) Sections of $\rho_{x x}$ near the moiré-induced satellite and main peak, for both the pristine and nanostructured graphene. The satellite peaks shows similar features as the main peaks, indicating that the substrate-induced moiré superlattice has cloned the engineered band structure. This is further shown in (B), where it is seen that the magnetoresistance of both main and moiré peaks increases in the pristine graphene, while it decreases for both peaks of the nanostructured graphene. A negative magnetoresistance is expected for the nanostructured graphene, the increasing magnetic field closes the band gap. The fact that a negative magnetoresistance is also seen at the moiré peak is a further indication that the engineered band structure is cloned by the substrate-induced moiré interaction.

able route towards true band structure engineering and sophisticated manipulation of the quantum transport behaviour of graphene devices. The realisation that nanolithography on the scale of the Fermi wavelength is possible without compromising graphene's excellent transport properties represents a significant breakthrough in the decade-long effort to realise the many exciting theoretical predictions based on "perfectly" nanostructured materials. Even the most delicate features, e.g. effects due to the moiré interference at interfaces, are preserved by the techniques described here. Furthermore, the possibility of combining nanostructuring with moiré superlattice effects in graphene, opens up for exciting possibilities in atomic scale band structure engineering of $2 \mathrm{D}$ materials.

\section{METHODS}

\section{Device fabrication}

The vdW heterostructures used in this study were fabricated using the so-called hot pick-up technique [31], based on Ref. [15], and the technique is described in detail in the Supplementary Information. The device consists of hBN encapsulated graphene situated on a graphite back gate, where the top and bottom hBN are $6 \mathrm{~nm}$, and $30 \mathrm{~nm}$ thick, respectively. After vdW stacking, the device is shaped into a multi-terminal Hall-bar and contacted using $\mathrm{Cr} / \mathrm{Au}(5 \mathrm{~nm} / 50 \mathrm{~nm})$ edge contacts [15]. Both of these steps are performed using standard EBL techniques. After metallisation and lift-off, all the terminals of the Hall-bar are electrically characterised at room temperature to ensure that the sample is homogeneous and of high quality.
For the nanostructured graphene, single-shot EBL was used to define a triangular array of holes with a center-tocenter distance of $35 \mathrm{~nm}$, estimated diameter of $20-23 \mathrm{~nm}$, and minimum feature sizes of $w=12-15 \mathrm{~nm}$. Due to a consistent etch angle of $\approx 66^{\circ}$ in hBN (see Supplementary Information) it is critical to have a thin top hBN crystal in order to achieve a successful pattern transfer into the underlying graphene.

\section{Electrical characterisation}

All electrical measurements were performed using either standard lock-in techniques $(17.77 \mathrm{~Hz}$ and $30 \mathrm{nA}$ source current), or using Keithley 2182a nanovoltmeters with a Keithley 6221 sourcing a current of $30 \mathrm{nA}$ in deltamode. Magnetotransport was performed in an Oxford TeslatronPT, with a base temperature of $1.5 \mathrm{~K}$. Unless stated, all temperatures were $4 \mathrm{~K}$.

\section{Ensemble-averaged tight-binding simulations}

The tight-binding simulations were carried out exactly the same way as in Ref. [36], and briefly outlined here. In the nearest-neighbor orthogonal tight-binding model, the Hamiltonian can be written as

$$
\hat{H}=\sum_{\langle i, j\rangle} t_{i j} \hat{c}_{i}^{\dagger} \hat{c}_{j}
$$

where $\hat{c}_{i}$ and $\hat{c}_{j}^{\dagger}$ are creation and annihilation operators, respectively, and $\langle i, j\rangle$ denotes nearest neighbours. The hopping parameter is $t_{i j}=t e^{i \phi_{i j}}$, where $\phi_{i j}$ is the Peierls 
phase, which takes into account the magnetic field, and $t=-3.033 \mathrm{eV}$ is the zero-field hopping parameter. The Peierls phase is given by $\phi_{i j}=(e / \hbar) \int_{\mathbf{r}_{i}}^{\mathbf{r}_{j}} \mathbf{A} \cdot d \mathbf{l}$, where $\mathbf{A}$ is the vector potential, and $\mathbf{r}_{i}$ is the position of atom $i$. The magnetic field in the leads is taken to be zero, which means that the vector potential in the Landau gauge is given by $\mathbf{A}(\mathbf{r})=\hat{\mathbf{y}} B \bar{x}$, where $\bar{x}=x \theta(x)-(x-d) \theta(x-d)$, where $\theta$ is the Heaviside step function and $d$ is the width of the barrier region. In this gauge, the Peierls phase becomes $\phi_{i j}=\frac{e B}{2 \hbar}\left(y_{j}-y_{i}\right)\left(\bar{x}_{i}+\bar{x}_{j}\right)$.

The conductance of the system is given by the Landauer-Büttiker formula $G=\frac{2 e^{2}}{h} T$, where $T=$ $\operatorname{Tr}\left\{\Gamma_{L} G^{\dagger} \Gamma_{R} G\right\}$ is the transmittance. We use the recursive Green's function method outlined in Refs. [45, 46] to extract the relevant elements of the retarded Green's function $G=\left((E+i \varepsilon) I-H-\Sigma_{L}-\Sigma_{R}\right)^{-1}$, where $H$ is the Hamiltonian matrix, and $\Sigma_{L / R}$ are the self-energies of the left and right leads. A small imaginary factor $i \varepsilon=-i t 10^{-4}$ is added for numerical stability.

Since our structures are periodic in the $y$-direction, any given crystal structure will lead to delicate fractaltype features, unique to the given atomic configuration. However, these features can effectively be suppressed by averaging over several unique atomic configurations, as we show in the Supplementary Information.

\section{Analytical Dirac ring model}

The Hamiltonian of this model is given by [6]

$$
\hat{H}=\left[\begin{array}{cc}
M(r) & v_{F} \Pi_{\mp} \\
v_{F} \Pi_{ \pm} & -M(r)
\end{array}\right] .
$$

Here, upper and lower signs correspond to $K$ and $K^{\prime}$ valleys, respectively. Also, $\Pi_{ \pm}=\Pi_{x} \pm i \Pi_{y}$ with $\boldsymbol{\Pi}=\mathbf{p}+$ $e \mathbf{A}$ the generalised momentum in a magnetic field $\mathbf{B}=$ $\nabla \times \mathbf{A}, v_{F}$ is the Fermi velocity, and $M(r)$ is a radially varying mass-term used to confine the Dirac fermions to a ring-shaped region, with inner and outer radius $R_{1}$, $R_{2}$, and width $w=R_{2}-R_{1}$. In particular, $M(r)=0$ inside the Dirac rings $\left(R_{1} \leq r \leq R_{2}\right)$, and $M(r)=\infty$ anywhere else. The full eigenvalue equation is given in the Supplementary Information.

\section{ACKNOWLEDGEMENTS}

We thank Gaetano Calogero, Johannes Handberg Juul Martiny, Kristen Kaasbjerg, and Aske Gejl for discussions. The Center for Nanostructured Graphene (CNG) is sponsored by the Danish National Research Foundation, Project DNRF103. B.S.J., L.G., J.C., and P.B. acknowledges funding from the EU Horizon 2020 'Graphene Flagship' 696656. T.P.G. and M.R.T. also acknowledge support for the VKR center of excellence QUSCOPE by the Villum foundation. D.M.A.M. acknowledges Villum Fonden project no. VKR023117 and EC Graphene
FET Flagship contract number 785219. K.W. and T.T. acknowledge support from the Elemental Strategy Initiative conducted by the MEXT, Japan, JSPS KAKENHI Grant Numbers JP18K19136 and the CREST (JPMJCR15F3), JST.

\section{AUTHOR CONTRIBUTIONS}

B.S.J. and L.G. conceived of the project, performed device fabrication, and transport measurements. B.S.J, L.G., and P.B. analysed the transport data. D.M.A.M performed and analysed the COMSOL simulations. J.C. and D.M.A.M. advised on measurements. J.D.T, E.D. and T.J.B. assisted with device fabrication. M.R.T. and T.G.B. performed simulations and developed the analytical model. K.W. and T.T. synthesised the hBN crystals. P.B. and A-P.J advised on the project. B.S.J., L.G., AP.J. and P.B. wrote the manuscript in consultation with all other authors.

\section{COMPETING INTERESTS}

The authors declare no competing interests.

\section{DATA AVAILABILITY}

The data that support the plots within this paper and other findings of this study are available from the corresponding author upon reasonable request. 
[1] Pedersen, T. G. et al. Graphene antidot lattices: designed defects and spin qubits. Phys. Rev. Lett. 100, 136804 (2008).

[2] Pedersen, T. G. et al. Optical properties of graphene antidot lattices. Phys. Rev. B 77, 245431 (2008).

[3] Gunst, T., Markussen, T., Jauho, A.-P. \& Brandbyge, M. Thermoelectric properties of finite graphene antidot lattices. Phys. Rev. B 84, 155449 (2011).

[4] Vanević, M., Stojanović, V. M. \& Kindermann, M. Character of electronic states in graphene antidot lattices: Flat bands and spatial localization. Phys. Rev. B $\mathbf{8 0}$, 045410 (2009).

[5] Nikitin, A. Y., Guinea, F. \& Martín-Moreno, L. Resonant plasmonic effects in periodic graphene antidot arrays. Applied Physics Letters 101, 151119 (2012).

[6] Thomsen, M. R. \& Pedersen, T. G. Analytical dirac model of graphene rings, dots, and antidots in magnetic fields. Phys. Rev. B 95, 235427 (2017).

[7] Pedersen, J. G., Gunst, T., Markussen, T. \& Pedersen, T. G. Graphene antidot lattice waveguides. Physical Review B 86, 245410 (2012).

[8] Dean, C. et al. Hofstadter's butterfly and the fractal quantum hall effect in moiré superlattices. Nature 497, 598 (2013).

[9] Ponomarenko, L. et al. Cloning of dirac fermions in graphene superlattices. Nature 497, 594 (2013).

[10] Hunt, B. et al. Massive dirac fermions and hofstadter butterfly in a van der waals heterostructure. Science $\mathbf{3 4 0 ,}$ 1427-1430 (2013).

[11] Forsythe, C. et al. Band structure engineering of 2d materials using patterned dielectric superlattices. Nature nanotechnology 13, 566-571 (2018).

[12] Caridad, J. M., Connaughton, S., Ott, C., Weber, H. B. \& Krstić, V. An electrical analogy to mie scattering. Nature communications 7, 12894 (2016).

[13] Stampfer, C. et al. Energy gaps in etched graphene nanoribbons. Phys. Rev. Lett. 102, 056403 (2009).

[14] Banszerus, L. et al. Ballistic transport exceeding 28 $\mu \mathrm{m}$ in cvd grown graphene. Nano letters 16, 1387-1391 (2016).

[15] Wang, L. et al. One-dimensional electrical contact to a two-dimensional material. Science 342, 614-617 (2013).

[16] Zeng, Y. et al. Ultra-high quality magnetotransport in graphene using the edge-free corbino geometry. arXiv preprint arXiv:1805.04904 (2018).

[17] Polshyn, H. et al. Quantitative transport measurements of fractional quantum hall energy gaps in edgeless graphene devices. arXiv preprint arXiv:1805.04199 (2018).

[18] Barone, V., Hod, O. \& Scuseria, G. E. Electronic structure and stability of semiconducting graphene nanoribbons. Nano letters 6, 2748-2754 (2006).

[19] Sandner, A. et al. Ballistic transport in graphene antidot lattices. Nano Lett. 15, 8402-8406 (2015).

[20] Yagi, R. et al. Ballistic transport in graphene antidot lattices. Phys. Rev. B 92, 195406 (2015).

[21] Han, M. Y., Brant, J. C. \& Kim, P. Electron transport in disordered graphene nanoribbons. Physical review letters 104, 056801 (2010).

[22] Fang, T., Konar, A., Xing, H. \& Jena, D. Mobility in semiconducting graphene nanoribbons: Phonon, impu- rity, and edge roughness scattering. Physical Review $B$ 78, 205403 (2008).

[23] Yang, Y. \& Murali, R. Impact of size effect on graphene nanoribbon transport. IEEE Electron Device Letters 31, 237-239 (2010).

[24] Bang, K. et al. Effect of ribbon width on electrical transport properties of graphene nanoribbons. Nano convergence 5, 7 (2018).

[25] Kim, M., Safron, N. S., Han, E., Arnold, M. S. \& Gopalan, P. Fabrication and characterization of largearea, semiconducting nanoperforated graphene materials. Nano Lett. 10, 1125-1131 (2010).

[26] Mackenzie, D. M. et al. Graphene antidot lattice transport measurements. Int. J. Nanotech. 14, 226-234 (2017).

[27] Mackenzie, D. M. et al. Batch fabrication of nanopatterned graphene devices via nanoimprint lithography. Appl. Phys. Lett. 111, 193103 (2017).

[28] Wang, M. et al. Cvd growth of large area smooth-edged graphene nanomesh by nanosphere lithography. Sci. Rep. 3, 1238 (2013).

[29] Peters, E., Giesbers, A., Zeitler, U., Burghard, M. \& Kern, K. Valley-polarized massive charge carriers in gapped graphene. Phys. Rev. B 87, 201403 (2013).

[30] Pan, J. et al. Berry curvature and nonlocal transport characteristics of antidot graphene. Phys. Rev. X 7, 031043 (2017).

[31] Pizzocchero, F. et al. The hot pick-up technique for batch assembly of van der waals heterostructures. Nat. Commun. 7, 11894 (2016).

[32] Terrés, B. et al. Size quantization of dirac fermions in graphene constrictions. Nature communications $\mathbf{7}, 11528$ (2016).

[33] Power, S. R., Thomsen, M. R., Jauho, A.-P. \& Pedersen, T. G. Electron trajectories and magnetotransport in nanopatterned graphene under commensurability conditions. Phys. Rev. B 96, 075425 (2017).

[34] Drienovsky, M. et al. Commensurability oscillations in one-dimensional graphene superlattices. Phys. Rev. Lett. 121, 026806 (2018).

[35] Pedersen, J. G. \& Pedersen, T. G. Hofstadter butterflies and magnetically induced band-gap quenching in graphene antidot lattices. Phys. Rev. B 87, 235404 (2013).

[36] Thomsen, M. R., Power, S. R., Jauho, A.-P. \& Pedersen, T. G. Magnetic edge states and magnetotransport in graphene antidot barriers. Phys. Rev. B 94, 045438 (2016).

[37] Heydrich, S. et al. Scanning raman spectroscopy of graphene antidot lattices: Evidence for systematic p-type doping. Applied Physics Letters 97, 043113 (2010).

[38] Sarma, S. D. \& Hwang, E. Two-dimensional metalinsulator transition as a strong localization induced crossover phenomenon. Phys. Rev. B 89, 235423 (2014).

[39] Radisavljevic, B. \& Kis, A. Mobility engineering and a metal-insulator transition in monolayer $\operatorname{mos}_{2}$. Nat. Mater. 12, 815 (2013).

[40] Shimizu, T. et al. Large intrinsic energy bandgaps in annealed nanotube-derived graphene nanoribbons. Nature nanotechnology 6, 45 (2011). 
[41] Gorbachev, R. et al. Detecting topological currents in graphene superlattices. Science 346, 448-451 (2014).

[42] Yankowitz, M. et al. Emergence of superlattice dirac points in graphene on hexagonal boron nitride. Nature Physics 8, 382 (2012).

[43] Zhang, Y., Tan, Y.-W., Stormer, H. L. \& Kim, P. Experimental observation of the quantum hall effect and berry's phase in graphene. Nature 438, 201 (2005).

[44] Novoselov, K. S. et al. Room-temperature quantum hall effect in graphene. Science 315, 1379-1379 (2007).

[45] Svizhenko, A., Anantram, M., Govindan, T., Biegel, B. \& Venugopal, R. Two-dimensional quantum mechanical modeling of nanotransistors. J. Appl. Phys. 91, 23432354 (2002).

[46] Lewenkopf, C. H. \& Mucciolo, E. R. The recursive green's function method for graphene. J. Comput. Electron. 12, 203-231 (2013).

[47] Weiss, D., Klitzing, K. V., Ploog, K. \& Weimann, G. Magnetoresistance oscillations in a two-dimensional elec- tron gas induced by a submicrometer periodic potential. EPL (Europhysics Letters) 8, 179 (1989).

[48] Park, C.-H., Yang, L., Son, Y.-W., Cohen, M. L. \& Louie, S. G. New generation of massless dirac fermions in graphene under external periodic potentials. Phys. Rev. Lett. 101, 126804 (2008).

[49] Kindermann, M., Uchoa, B. \& Miller, D. L. Zero-energy modes and gate-tunable gap in graphene on hexagonal boron nitride. Physical Review B 86, 115415 (2012).

[50] Wallbank, J., Patel, A., Mucha-Kruczyński, M., Geim, A. \& Fal'ko, V. Generic miniband structure of graphene on a hexagonal substrate. Physical Review B 87, 245408 (2013).

[51] Lee, M. et al. Ballistic miniband conduction in a graphene superlattice. Science 353, 1526-1529 (2016). 


\title{
Supplementary Information: Lithographic band structure engineering of graphene
}

\author{
Bjarke S. Jessen, Lene Gammelgaard, Morten R. Thomsen, David M. A. Mackenzie, \\ Joachim D. Thomsen, José M. Caridad, Emil Duegaard, Kenji Watanabe, Takashi Taniguchi, \\ Timothy J. Booth, Thomas G. Pedersen, Antti-Pekka Jauho, and Peter Bøggild
}

\section{CONTENTS}

1. Fabrication

1.1. vdW Stacking

1.2. Graphene to hBN etch selectivity

1.3. hBN etch angle and edge-roughness

1.3.1. $\mathrm{hBN}$ etch angle

1.3.2. Reducing edge-roughness

1.4. Nanostructuring

2. Calculations

2.1. Tight-binding simulations

2.2. Analytical Dirac ring calculations

2.3. Gapped carrier density

3. Supporting Text

3.1. Moiré superlattice effects

3.2. Extraction of half-gaps from Arrhenius plot

3.3. Zero-field half-gap estimation of the nanostructured region

3.4. Additional magnetotransport data of the pristine and nanopatterned areas

3.5. Investigation of non-zero $\rho_{x y}$ at $B=0 \mathrm{~T}$ 3.5.1. Transport data

3.5.2. COMSOL study

References

1

1

2

3

3

3

3

3

3

4

7

7

7

7

9

9

9

9

9

12

\section{FABRICATION}

\section{1. vdW Stacking}

The vdW heterostructure stacking was performed with the hot pick-up technique [S1], based on the dry pick-up method of Ref. [S2], with slight modifications. As shown in Fig. S1, the hot pick-up technique has the following steps:

1. Pick-up: A glass-slide with a block of poly(propylene carbonate) (PPC) (CAS number 25511-85-7) coated polydimethylsiloxane (PDMS) (SYLGARD®184, 10:1 base:curing), is used to pick and place the crystals*. A crystal is picked up by approaching the crystal with the

\footnotetext{
* The PDMS is glued to the glass-slide using epoxy, and is gently plasma cleaned $\left(\mathrm{O}_{2}\right)$ immediately prior to applying the PPC.
}

PDMS/PPC at a temperature of $55^{\circ} \mathrm{C}$, where the PPC becomes soft and easily conforms to the crystal. The sample is then cooled down to $40^{\circ} \mathrm{C}$, where the PPC is sufficiently rigid to pick up the crystal when the glass slide is (quickly) retracted.

2. De-wrinkle bake: Often the crystals will exhibit folds and wrinkles after pick-up, severely limiting the usable areas. This can easily be remedied by a soft de-wrinkle bake of the glass slide, as seen in Fig. S1. Care should be taken to not heat more than necessary, as the crystals will tend to start folding back onto themselves - this is especially the case for thin crystals (sub-10 nm). Folding can be seen on the top corner of the hexagonal boron nitride (hBN) crystal in the lower panel of Fig. S1. It is thus a good idea to do the de-wrinkle bake under a microscope, to make sure folding is not too severe.

3. Drop-down: The crystal on the PDMS/PPC is aligned to a target flake. By increasing the temperature of the target sample, the adsorbed water and hydrocarbons become highly mobile, allowing for the gradual contact between the crystals to squeeze out any adsorbed contamination. The target sample is heated to $\sim 110^{\circ} \mathrm{C}$ when the crystal on the PDMS/PPC block is brought in contact slowly. As full contact is achieved, the temperature is lowered to $70^{\circ} \mathrm{C}$. At this temperature the $\mathrm{PPC}$ is soft enough to be peeled off from the crystals when the glass-slide is slowly retracted, while remaining on the PDMS. It is thus possible to do a carefully controlled "drop-down" of the crystal.

4. Adhesion bake: The stack of crystals on the target chip is baked at $170^{\circ} \mathrm{C}$ to increase the adhesion between the stacked crystals, relative to the $\mathrm{SiO}_{2}$ substrate, before picking them up to continue the vdW heterostructure assembly according to step 1 .

The stack is assembled from the top and down, meaning that the first crystal that is picked-up is the top hBN which then is dropped-down on the graphene. Graphene and $\mathrm{hBN}$ is then picked up and dropped down on the bottom hBN. Finally, the hBN encapsulated graphene is picked up and dropped down on the graphite back gate, repeating the four steps three times. 
a
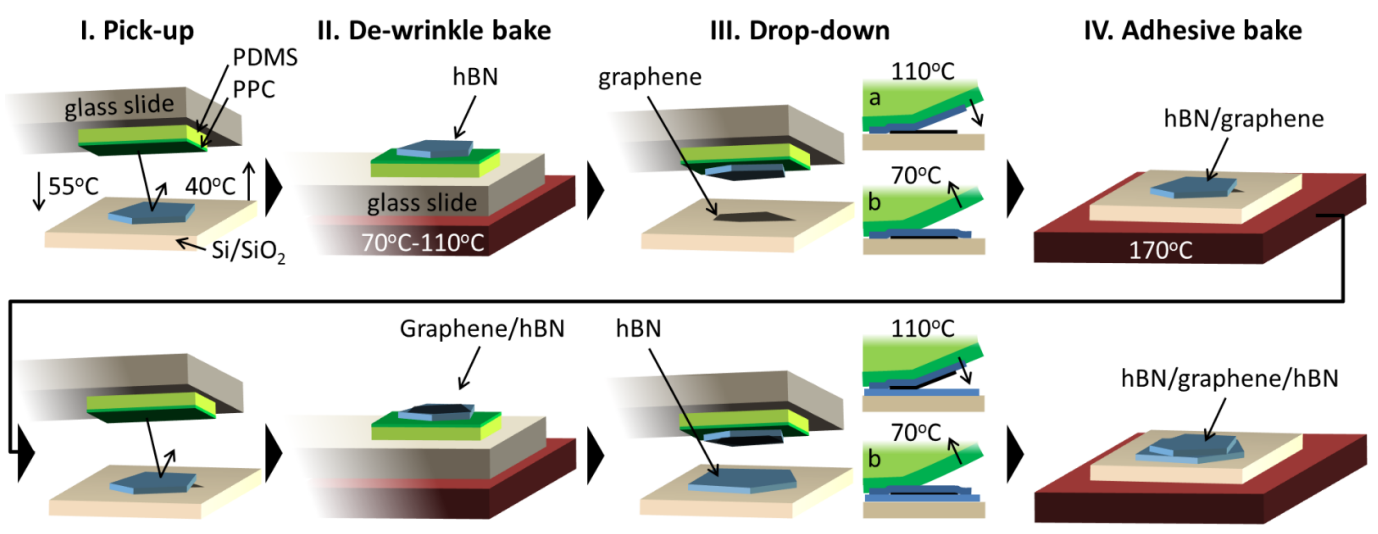

b

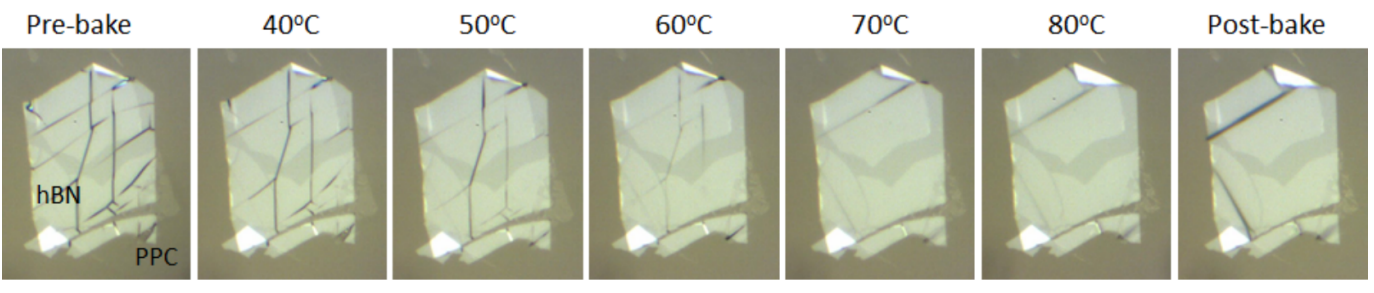

FIG. S1. Preparation of van der Waals heterostructures: (a) Schematic outline of the hot pick-up technique for assembly of van der Waals heterostructures. PPC is brought in contact with the target $\mathrm{hBN}$ crystal at $55^{\circ} \mathrm{C}$, cooled to $40^{\circ} \mathrm{C}$, and the $\mathrm{hBN}$ is picked up. If wrinkles appear in the crystal after being picked up, these can be straightened out by baking the PPC+crystals at elevated temperatures $\left(70^{\circ} \mathrm{C}-110^{\circ} \mathrm{C}\right)$. The $\mathrm{hBN}$ is now brought in contact with the next crystal, e.g. graphene. By raising the temperature to $110^{\circ} \mathrm{C}$ during contact, adsorbed species on the crystals become highly mobile, thus making it easier to squeeze them away as the crystals are gradually put in contact. When in full contact the hBN is "dropped down" on the graphene, by slowly retracting the $\mathrm{PPC}$ at $70^{\circ} \mathrm{C}$. The sample can now be baked on a hot-plate at $170^{\circ} \mathrm{C}$ for 30 minutes or more, to promote adhesion between the crystals. The entire process can now be repeated, i.e. the hBN+crystal can be picked up, de-wrinkled, dropped down on another crystal, baked, and picked up. (b) De-wrinkle bake of hBN after pick-up. As the temperature is gradually increased, the PPC becomes soft enough for wrinkles in the hBN to be smoothed out.

\subsection{Graphene to $\mathrm{hBN}$ etch selectivity}

Due to the inclusion of a graphite back-gate, it is important that the bottom hBN dielectric, separating the device and the gate, is of high quality and not damaged by etching of the upper layers. For this reason, we have developed reactive ion etch (RIE) recipes, which are highly selective towards either graphene or hBN. In particular, we use a low power $\mathrm{SF}_{6}$ etch for etching of $\mathrm{hBN}$, which stops on the graphene layer, which is fluorinated in the process, and a low power $\mathrm{O}_{2} / \mathrm{Ar}$ etch which is perfectly selective between graphene and $\mathrm{hBN}$. This greatly eases fabrication compared to commonly used gases such as $\mathrm{CHF}_{3}$, which etches graphene, hBN, and $\mathrm{SiO}_{2}$ at approximately the same rate. Relevant etch-rates are given in Table S1, and all etching was performed in an SPTS Pro ICP system.

hBN etch parameters: For hBN etching, we use a gas of $\mathrm{SF}_{6}$ at $80 \mathrm{sccm}$, a relatively high pressure of $80 \mathrm{mTorr}$, and a forward power of $75 \mathrm{~W}$ at $13.56 \mathrm{MHz}$, with the coil of the ICP-RIE system turned off.

Graphene etch parameters: For graphene etching, we use a gas of $\mathrm{O}_{2} / \mathrm{Ar}$ at $5 / 15 \mathrm{sccm}$, a relatively high pressure of $80 \mathrm{mTorr}$, and a forward power of $20 \mathrm{~W}$ at $13.56 \mathrm{MHz}$, with the coil of the ICP-RIE system turned off.

Care should be taken to properly clean and precondition the plasma chamber prior to using these recipes, as residual gas species from other process runs can ruin the selectivity. While we stress that the selectivity and etchrates might be different in other etching systems, a guiding principle should be to use high pressure, low power, careful cleaning between etching steps, and no addition of $\mathrm{O}_{2}$ when etching the hBN.

TABLE S1. Reactive ion etching etch rates:. In contrast to the often used $\mathrm{CHF}_{3}$ gas, highly selective etching of $\mathrm{hBN}$ and graphene can be done with pure $\mathrm{SF}_{6}$ or $\mathrm{O}_{2} / \mathrm{Ar}$, respectively. Furthermore, $\mathrm{SF}_{6}$ imparts only low damage to the $\mathrm{SiO}_{2}$ substrate.

\begin{tabular}{lccc}
\hline Material & $\mathrm{CHF}_{3}(\mathrm{~nm} / \mathrm{min})$ & $\mathrm{O}_{2} / \mathrm{Ar}(\mathrm{nm} / \mathrm{min})$ & $\mathrm{SF}_{6}(\mathrm{~nm} / \mathrm{min})$ \\
\hline Graphene & 30 & 15 & 0 \\
hBN & 30 & 0 & 400 \\
$\mathrm{PMMA}$ & 30 & 80 & 31 \\
$\mathrm{SiO}_{2}$ & 30 & 0 & $<7$ \\
\hline
\end{tabular}




\section{3. hBN etch angle and edge-roughness}

\subsection{1. $h B N$ etch angle}

The hBN etch angle and consistency is of great importance for a successful pattern transfer and fabrication planning, especially in the case of ultra-dense nanopatterning, as it turns out. If the angle is too shallow, the intended structure will not be successfully transferred to the underlying graphene, while an inconsistent etch angle will lead to increased edge roughness. Figure S2 shows the measured etch angle, $\phi$, of lines in $\mathrm{hBN}$, relative to vertical, spanning 90 degrees of in-plane angles, covering all crystallographic directions of $\mathrm{hBN}$.

We measure the etch angle in $\mathrm{hBN}$ to be $66.05^{\circ} \pm 0.65^{\circ}$ and independent of the lattice orientation of the $\mathrm{hBN}$ crystal, which leads to smooth edges in circular structures. The geometric relation between the diameter of the (designed) hole on the surface of the top hBN, $D$, and the diameter of the (actual) hole in the graphene, $d$, is

$$
d=D-2 h \cot (\phi)
$$

where $h$ is the thickness of the $\mathrm{hBN}$ and $\phi$ is the etch angle, as shown schematically in Fig. S2.

\subsubsection{Reducing edge-roughness}

Even anisotropic etching of dense nanostructures in graphene has been shown to result in edge-roughness and defected graphene on the scale of $1.5 \mathrm{~nm}$ to $3 \mathrm{~nm}$ [S3]. If our samples display the same level of roughness, the triangular lattice with $35 \mathrm{~nm}$ period and $21 \mathrm{~nm}$ diameter will result in more than $20 \%$ of the remaining graphene being defected. To avoid such levels of disorder, the etching should thus be quite gentle while being able to transfer the intended pattern to the graphene successfully. Since the etching recipes presented here are highly selective between $\mathrm{hBN}$ and graphene, it is only necessary to improve the performance of the hBN etch.

During testing and optimization of our processes, we consistently saw increased disorder in nanostructured $\mathrm{hBN}$ where the holes completely punch through the crystal. Under identical process conditions, thick hBN crystals, or samples where the selective $\mathrm{SF}_{6}$ etch terminates on the graphene, were consistently well-defined compared to thinner $\mathrm{hBN}$ where the etch punched through to the $\mathrm{SiO}_{2}$ substrate. This is shown in Fig. S3a-b, which shows two samples fabricated with the same process parameters in the same process run, but where the etch in Fig. S3a went all the way through the crystal (hBN thickness $<15 \mathrm{~nm}$ ), while the etch in Fig. S3b did not penetrate all the way (hBN thickness $>50 \mathrm{~nm})$. Similarly, we see much lower edge-roughness when etching our vdW heterostructures, where we use the selective
$\mathrm{SF}_{6}$ etch to only etch the top hBN, as shown in Fig. S3d.

\subsection{Nanostructuring}

Electron beam lithography was carried out in a $100 \mathrm{keV}$ JEOL JBX-9500FS system, with the positive e-beam resist polymethyl methacrylate (PMMA) as an etch mask. We use a $2 \%$ wt solution of 996K PMMA from SigmaAldrich, dissolved in anisole and spin-coated to a $50 \mathrm{~nm}$ thick layer on the device. A thin layer is used to minimize forward scattering and ensure a well-defined patterntransfer.

We found that the best results, regarding resolution and low roughness, were obtained using single-shot exposures for the nanostructures. Doses are thus reported in units of $\mathrm{fC} /$ dot instead of the conventional $\mu \mathrm{C} / \mathrm{cm}^{2}$. Various developers for the PMMA were tested, including; IPA, ethanol, IPA: $\mathrm{H}_{2} \mathrm{O}$ (3:1), ethanol: $\mathrm{H}_{2} \mathrm{O}$ (3:1). IPA: $\mathrm{H}_{2} \mathrm{O}$ was tested at both $-17^{\circ} \mathrm{C}$ and $20^{\circ} \mathrm{C}$. IPA and ethanol at $20^{\circ} \mathrm{C}$ yielded similar results, with the highest pattern-density and widest process latitudes, and IPA was thus used for fabrication of the nanopatterning presented in the main text.

Figure $\mathrm{S} 4$ shows the process latitude for periods of $30 \mathrm{~nm}$ to $50 \mathrm{~nm}$ triangular lattices, along with the diameter versus dose for the $50 \mathrm{~nm}$ period. We find similar diameter vs dose relations for all our nanostructures in hBN.

\section{CALCULATIONS}

\subsection{Tight-binding simulations}

Tight-binding simulations were made for a pattern with roughly 4 times smaller dimensions than in the nanostructured region of the experimental device, namely with a center-to-center distance of $35 a \simeq 8.61 \mathrm{~nm}$ and round holes with radius $R=9 a \simeq 2.21 \mathrm{~nm}$. Scaling rules are used to convert the simulation result to the equivalent result of a larger structure: By scaling all lengths with a factor $a$, the energy becomes $E / a$ and the magnetic field becomes $B / a^{2}$, in accordance with [S4]. We take four rows of holes in the transport (armchair) direction and use periodic boundary conditions in the perpendicular direction. Also, the conversion factor between gate voltage, $V_{G}$, and energy was determined by fitting against Landau levels of the pristine part of the spectrum and gave rise to $V_{G}=125 \frac{\mathrm{V}}{\mathrm{eV}} E^{2}$. The exact value of the radius does not seem to play an important role for the Landau level features. Furthermore, the type and strength of the disorder also seems to play only a minor role. Since our structures are periodic in the $y$-direction, any given crystal structure will lead to delicate fractal-type features, unique to the given atomic configuration. However, the these features can effectively be suppressed by averaging 
a

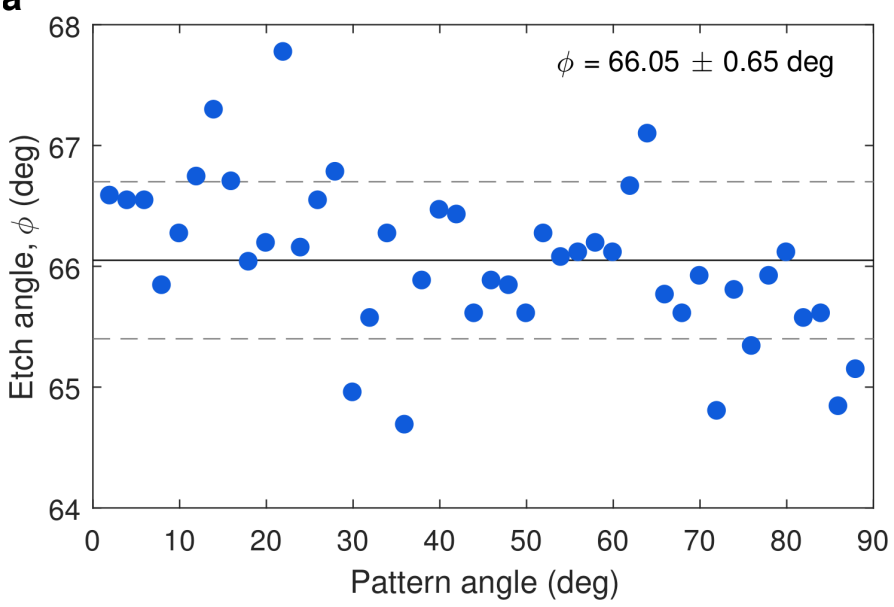

b
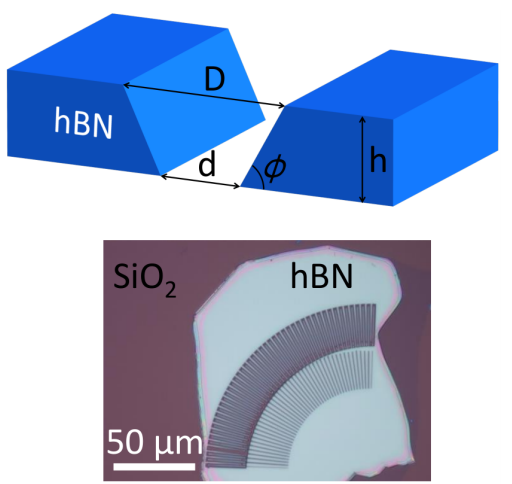

FIG. S2. Etch angle of hexagonal boron nitride: (a) Etch angle of hBN relative to horizontal, $\phi$, as a function of the angle between the etch pattern and the hBN lattice. The etch angle is seen to be independent of the orientation relative to the hBN crystallographic direction. (b) Optical micrograph of the etched hBN flake and a sketch of the angles influence on pattern-transfer through a hBN flake.

a

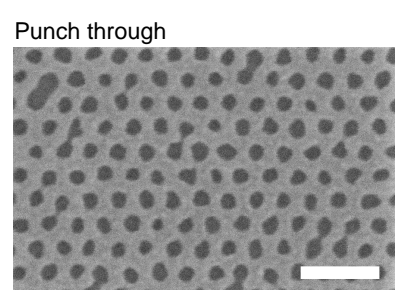

b

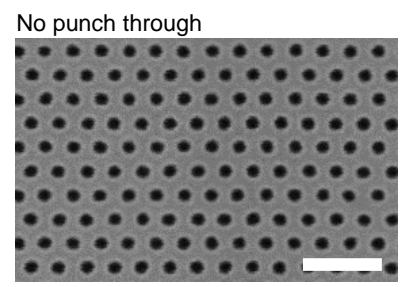

C

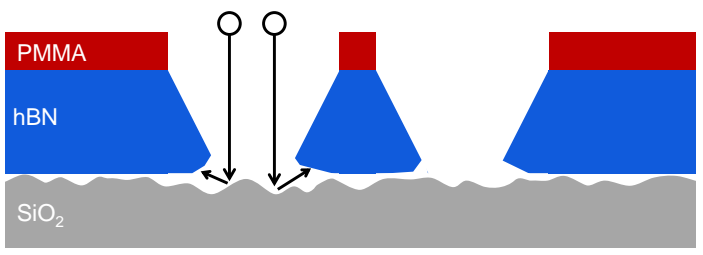

d

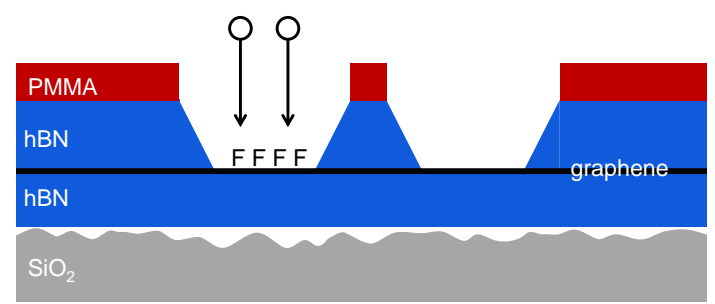

FIG. S3. Reducing edge-roughness during reactive ion etching: SEM images of a hexagonal lattice with a period of $35 \mathrm{~nm}$ etched into $(\mathbf{a})$ a thin $\mathrm{hBN}(<15 \mathrm{~nm})$ and $(\mathbf{b})$ a thick flake of hBN $(>50 \mathrm{~nm})$. Scale bars are $100 \mathrm{~nm}$. (c-d) shows schematics of the proposed etch dynamics in the two situations of a punch through and no punch through.

over several unique atomic configurations, as we show in Fig. S5. We incorporate the effects of small amounts of edge-disorder by allowing for random removal of the inner-most edge atoms around each hole, with a certain probability. For each presented magnetotransport calculation, this procedure is repeated 10 times, resulting in ensemble-averaged spectra. For a chance of $5 \%$ and higher to remove an edge atom, we recover most of the features of the experimental results.

\subsection{Analytical Dirac ring calculations}

The Dirac model is described in Ref. S5, and will be briefly outlined here. A graphene ring with inner and outer radius $R_{1}$ and $R_{2}$ is considered in a perpendicular magnetic field. The Dirac equation is given by $H \Psi=$ $E \Psi$ with wave function spinor $\Psi=\left(\psi_{A}, \psi_{B}\right)^{T}$ and the Hamiltonian $H=(\hat{\mathbf{p}}+e \mathbf{A}) \cdot\left(\sigma_{x}, \sigma_{y}\right)^{T}+M(r) \sigma_{z}$, where $\sigma_{x, y, z}$ are Pauli matrices, $\hat{\mathbf{p}}$ is the momentum operator, $\mathbf{A}$ is the vector potential, and $M(r)$ is a piecewise constant mass term, which takes into account the confinement of electrons by the ring, with $M(r) \rightarrow \infty$ outside the ring and given by the moiré induced half-gap $\Delta_{m}$ inside. As the geometry has perfect circular symmetry, the spinor 

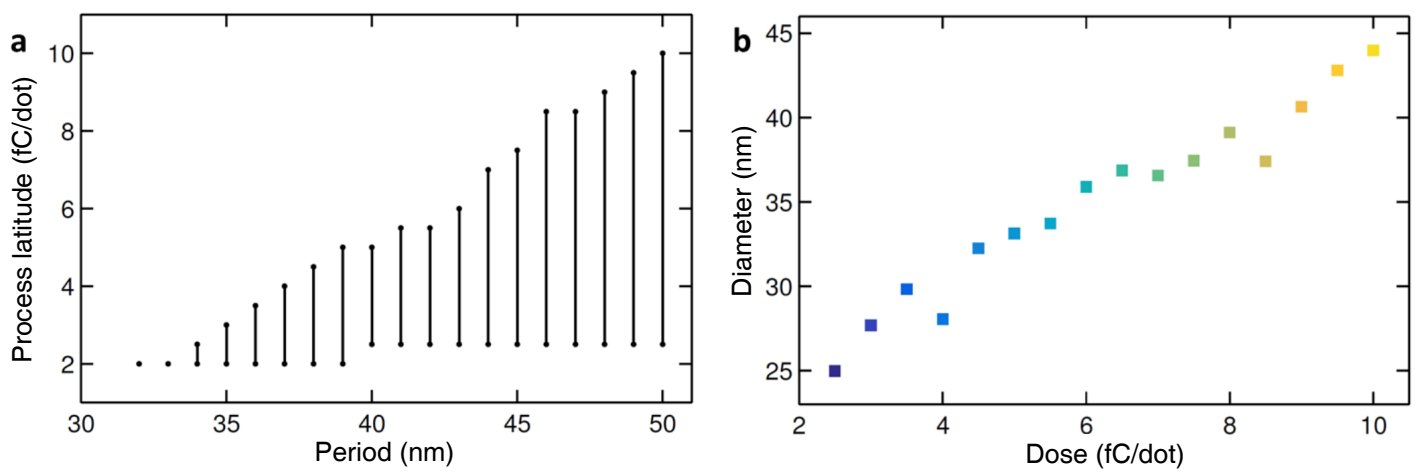

c

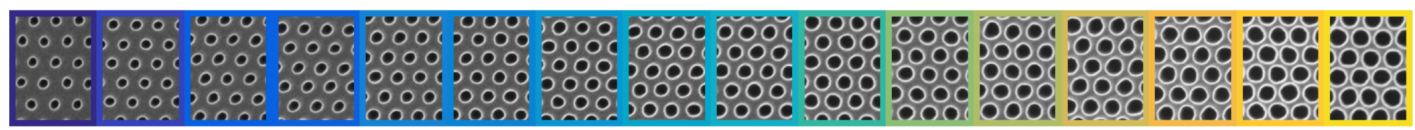

FIG. S4. Process lattitude and dose-dependant hole sizes: (a) Process latitude of the e-beam dose as a function of the period of a triangular lattice. For doses to be in the process latitude, all holes have to be present, and no holes may be merged within the $2 \mu \mathrm{m}$ by $2 \mu \mathrm{m}$ pattern area. (b) Diameter variation with increasing dose for the $50 \mathrm{~nm}$ period lattice. (c) Sections of SEM micrographs of the $50 \mathrm{~nm}$ period images used for the plot in (b), the images are colour framed to match the plot.

components can be written as a product of angular and radial parts,

$$
\psi_{A}(r, \phi)=f(r) e^{i m \phi},
$$

where $m$ is an integer quantum number describing the angular momentum of the state. A similar expression holds for $\psi_{B}$. The problem is then solved by requiring continuity of both spinor components on the $R_{1}$ and $R_{2}$ boundaries, which gives rise to an eigenvalue condition,

$$
\begin{aligned}
0= & \left\{U_{-\varepsilon^{2}}^{-m}\left(\rho_{2}^{2}\right)\left[\varepsilon L_{\varepsilon^{2}}^{-m-1}\left(\rho_{1}^{2}\right)-\rho_{1} L_{\varepsilon^{2}-1}^{-m}\left(\rho_{1}^{2}\right)\right]-\varepsilon L_{\varepsilon^{2}}^{-m-1}\left(\rho_{2}^{2}\right)\left[U_{-\varepsilon^{2}}^{-m}\left(\rho_{1}^{2}\right)+\rho_{1} \varepsilon U_{1-\varepsilon^{2}}^{1-m}\left(\rho_{1}^{2}\right)\right]\right. \\
& \left.+\rho_{2} \varepsilon U_{1-\varepsilon^{2}}^{1-m}\left(\rho_{2}^{2}\right)\left[\rho_{1} L_{\varepsilon^{2}-1}^{-m}\left(\rho_{1}^{2}\right)-\varepsilon L_{\varepsilon^{2}}^{-m-1}\left(\rho_{1}^{2}\right)\right]-\rho_{2} L_{\varepsilon^{2-1}}^{-m}\left(\rho_{2}^{2}\right)\left[U_{-\varepsilon^{2}}^{-m}\left(\rho_{1}^{2}\right)+\rho_{1} \varepsilon U_{1-\varepsilon^{2}}^{1-m}\left(\rho_{1}^{2}\right)\right]\right\} \\
& \times\left\{\varepsilon^{2} L_{\varepsilon^{2}}^{-m-1}\left(\rho_{1}^{2}\right) U_{1-\varepsilon^{2}}^{1-m}\left(\rho_{1}^{2}\right)+L_{\varepsilon^{2}-1}^{-m}\left(\rho_{1}^{2}\right) U_{-\varepsilon^{2}}^{-m}\left(\rho_{1}^{2}\right)\right\}^{-1},
\end{aligned}
$$

that is solved numerically. Here $\varepsilon=E /\left(\hbar \omega_{c}\right)$ is dimensionless energy, $\hbar \omega_{c}=\sqrt{2} \hbar v_{F} / l_{B}$ is the cyclotron energy, $\rho=r / \sqrt{2} l_{B}$ is the dimensionless radial coordinate, and $l_{B}=\sqrt{\hbar /(e B)}$ is the magnetic length. Furthermore, $U_{a}^{b}(z)$ is the confluent hypergeometric function of the second kind,

$$
\begin{aligned}
U_{a}^{b}(z)= & \frac{\Gamma(1-b)}{\Gamma(a+1-b)}{ }_{1} F_{1}(a ; b ; z) \\
& +\frac{\Gamma(b-1)}{\Gamma(a)} z^{1-b}{ }_{1} F_{1}(a+1-b ; 2-b ; z),
\end{aligned}
$$

where $\Gamma(n)$ is the Gamma function, $L_{a}^{b}(z)$ is the generalized Laguerre function,

$$
L_{a}^{b}(z)=\frac{\Gamma(b-1+a)}{\Gamma(b-1) a !}{ }_{1} F_{1}(-a ; b+1 ; z),
$$

and ${ }_{1} F_{1}(a ; b ; z)$ is the confluent hypergeometric function of the first kind,

$$
{ }_{1} F_{1}(a ; b ; z)=\frac{\Gamma(b)}{\Gamma(b-a) \Gamma(a)} \int_{0}^{1} e^{z t} t^{a-1}(1-t)^{b-a-1} d t .
$$

We stress that numerical evaluation of the hypergeometric functions is far from trivial, and we recommend the usage of the arbitrary-precision floating-point mpmath package for Python [S6], or equivalent implementations in Wolfram Mathematica.

In Fig. $\quad \mathrm{S} 6$ we show a selection of Dirac ring band structures for $10 \mathrm{~nm} \leqslant R_{1} \leqslant 25 \mathrm{~nm}$ and $30 \mathrm{~nm} \leqslant R_{2} \leqslant$ $45 \mathrm{~nm}$, with red (blue) bands corresponding to polarised states belonging to the $K\left(K^{\prime}\right)$ valley. Three prominent trends of the band structures can be seen: 


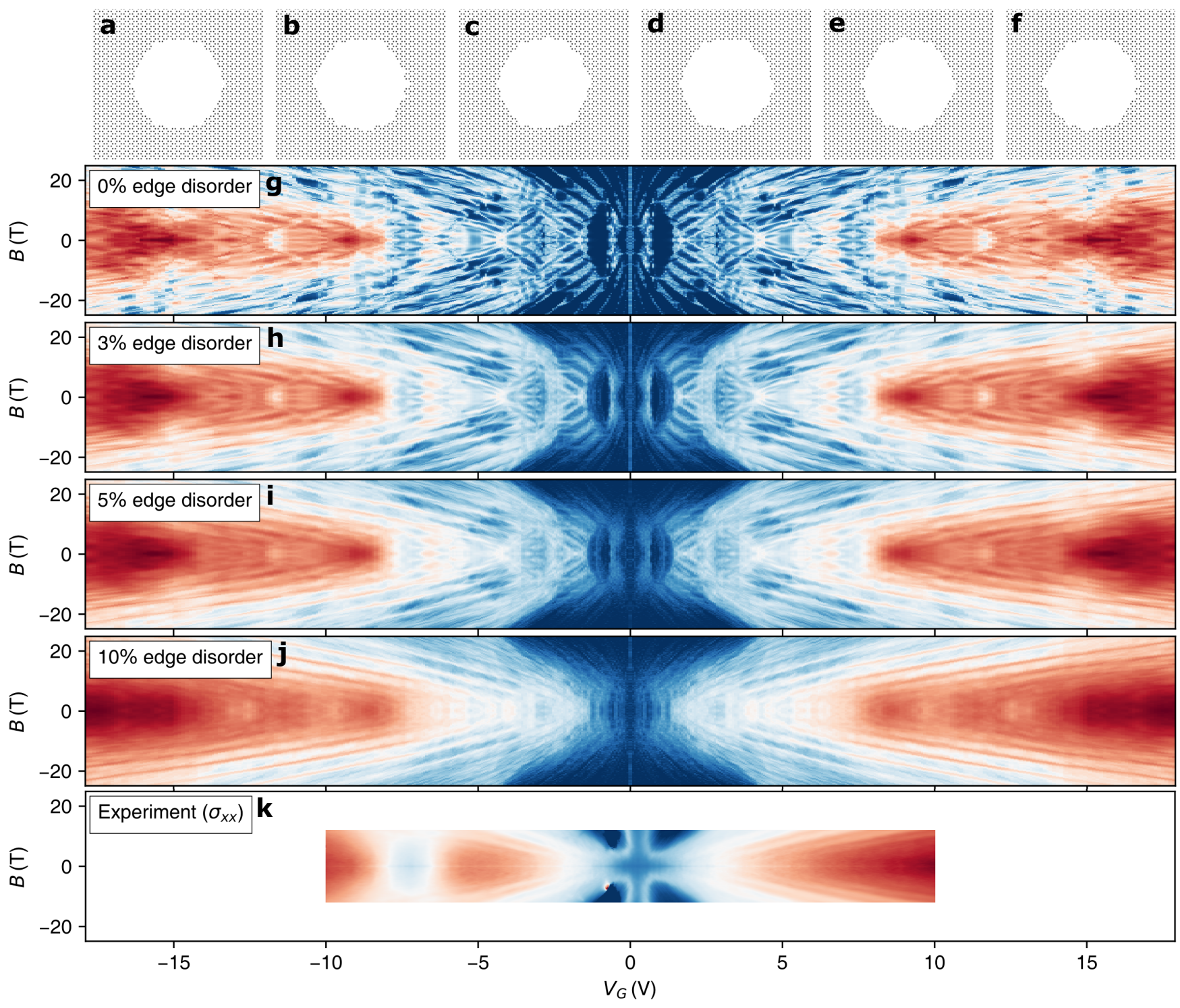

FIG. S5. Ensemble-averaging of different edge-configurations: (a-f) Different random configurations of edge disorder in our simulations. The disorder is introduced by randomly moving atoms on the very edge of the hole, followed by elimination of any dangling bonds. The following four panels shows ensemble-averaged conductance, $G_{x x}=\frac{2 e^{2}}{h} T_{x x}$, from tight-binding transport simulations at different degrees of disorder. (g-j) The four panels, from top to bottom, represents random removal of atoms on the edge of the holes with a probability of $0 \%, 3 \%, 5 \%$, and $10 \%$, respectively. The gate voltage, $V_{G}$, was determined by fitting Landau levels to the pristine graphene part, which gave rise to the conversion factor $V_{G}=125 \frac{V}{e V} E^{2}$, where $E$ is the energy used in the tight-binding models. (k) For comparison the experimental data is included.

(i) Rings with the same width, $w$, also possess the same $B=0 \mathrm{~T}$ half-gap, following

$$
E_{g} / 2=\Delta_{0}=1 \mathrm{eVnm} / w
$$

which also defines the spacing between subsequent bands. This is similar to the energy gap scaling seen in graphene nanoribbons [S7].

(ii) The inner radius, $R_{1}$, defines the spacing of the bands emerging towards $E=0$. This is similar to the semiclassical bands described in the main text and Ref. [S4], as well as the measured magnetoresistance oscillations, and corresponds to electronic states confined by a magnetic field around the inner perimeter.

(iii) At any finite magnetic field, the valley-degeneracy is lifted, with the conduction- and valence-band of the $K\left(K^{\prime}\right)$ valley moving up (down) in energy as the magnetic field is increased. This continually decreases the band gap, and when the magnetic length, $l_{B}$, i.e. the length-scale associated with localization of charged particles due to the magnetic field, is much smaller than the width of the rings the band gap is effectively closed. In other words, when the charge carriers are confined to 
length-scales much smaller than the geometric confinement, the band gap is closed, and the usual Landaulevels of single-layer graphene emerges. This effect has been theoretically predicted in Ref. [S8].

\subsection{Gapped carrier density}

While the Hall-measurement of the pristine area shows the expected linear relation between gate voltage and carrier density, the Hall-measurement of the Dirac ring area exhibits a gap in the carrier density, as shown in Fig. 2C of the main text. This behaviour is consistent with the absence of charge carriers in gapped graphene, with the expected carrier density given by

$$
n=\int_{-\infty}^{\infty}\left[f\left(E-E_{F}\right)-f(E)\right] D(E) d E .
$$

Here $f(E)$ is the Fermi function, $E_{F}$ is the Fermi energy, and we use the gapped graphene model for the density of states [S9], $D(E)=\frac{2|E|}{\hbar^{2} v_{F}^{2} \pi} \theta\left(E_{F}-\Delta\right)$, with the half-gap given by $\Delta=E_{g} / 2$ and $\theta$ is the Heaviside step function.

\section{SUPPORTING TEXT}

\subsection{Moiré superlattice effects}

In the pristine area of the device, we observe satellite peaks at $V_{G}=V_{0} \pm 7.55 \mathrm{~V}$ corresponding to a moiré period of $\lambda_{m, 1}=10.2 \mathrm{~nm}$, and at $V_{G}=V_{0}-2.65 \mathrm{~V}$ corresponding to a $\lambda_{m, 2}=17.2 \mathrm{~nm}$. $V_{0}$ is the gate voltage at the charge neutrality point. The $\lambda_{m, 1}$ peak is also present in the nanostructured area of the device.

The moiré period is calculated by [S10, S11]:

$$
\lambda_{m, 1}=\sqrt{\frac{8}{\sqrt{3} \Delta \alpha \Delta V_{G}}},
$$

where $\alpha$ is the lever arm constant relating the applied gate voltage to the number of induced charge carriers, as measured by Hall measurements. For the sample presented in the main text, $\alpha=5.87 \times 10^{11} \mathrm{~cm}^{-2} \mathrm{~V}^{-1}$. Given a twist angle, $\theta$, of the graphene relative to the underlying $\mathrm{hBN}$ substrate, the shortest possible moiré period is [S10]

$$
\lambda_{m, 1}=\frac{(1+\delta) a}{\sqrt{2(1+\delta)(1-\cos (\theta))+\delta^{2}}},
$$

where $\delta$ is the lattice mismatch of $1.8 \%$ for $\mathrm{hBN}$ and graphene, and $a$ is the lattice constant of graphene, $0.246 \mathrm{~nm}$. Given our data, $\lambda_{m, 1}=10.2 \mathrm{~nm}$ correspond to a twist angle of $\theta=0.9^{\circ}$. Given Eq. S3 the shortest possible moiré period for graphene on hBN never will exceed $\sim 14 \mathrm{~nm}$, and the moiré period of $17.2 \mathrm{~nm}$ can therefore not be explained by this simple picture.

Indications of moiré superlattices with longer periodicities have been observed in literature. In Ref. [S12] Chen et al. [S12] reports the emergence of $>14 \mathrm{~nm}$ periods, based on a slightly closer inspection of the hBNgraphene superlattice. The hBN-graphene superlattice has three main regions; one where the boron atoms are in the centre of the carbon hexagons of the graphene, one where the nitrogen atoms are in the centre of the carbon hexagons, and finally a region where the atoms in the graphene and the $\mathrm{hBN}$ lattice are on top of each other. The most commonly observed moiré pattern corresponds to the superlattice constructed of the two regions where the boron/nitrogen atoms are in the centre of the carbon hexagons, as shown by the blue and red regions connected by a white hexagon in Fig. S7. A secondary moiré pattern, corresponding to the superlattice constructed by the areas where the atoms in $\mathrm{hBN}$ and graphene are on top of each other, yield period a factor of $\sqrt{3}$ larger than the conventional moiré pattern, as illustrated with the orange hexagon in Fig. S7.

Another report of $>14 \mathrm{~nm}$ periods was recently presented by Wang et al. [S13], in devices where the graphene is aligned to both the top and bottom encapsulating $\mathrm{hBN}$. In particular, they found that the two superlattices from the aligned graphene can result in a third superlattice, with periods larger than $14 \mathrm{~nm}$, as evidenced from transport measurements.

While our measured $\lambda_{m, 2}$ of $17.2 \mathrm{~nm}$ is very close to $\sqrt{3} \cdot 10.2 \mathrm{~nm}=17.7 \mathrm{~nm}$ (cf. Chen et al.), it should be noted that according to Chen et al. higher period moiré patterns were shown to appear at higher energies, while we observe the larger wavelength pattern to appear at lower energies, i.e. in line with Wang et al.

\subsection{Extraction of half-gaps from Arrhenius plot}

Figure S8 shows the temperature dependency of the minimum conductance, $\sigma_{m i n}$, for the pristine and nanostructured regions of the device. From the Arrhenius relation, $\sigma \propto \exp \left[\Delta /\left(k_{B} T\right)\right]$, we can extract activation energies, $\Delta=E_{g} / 2$, from the linear parts of inverse temperature plotted against $\ln \left(\sigma_{\text {min }}\right)$, where $k_{B}$ is the Boltzmann constant. In systems with multiple activation energies, the higher activation energies dominate at higher temperatures while the lower activation energies dominate at lower temperatures [S14]. We extract one activation energy of $\Delta \approx 14 \pm 1 \mathrm{meV}$ in both the pristine and nanostructured regions, and one of $\Delta \approx 74 \pm 11 \mathrm{meV}$ which is only seen in the nanostructured region. The size of the smaller activation energy fits well with earlier reported moiré-induced half-gaps of graphene devices with similar small twist angles to the present work [S15], while 


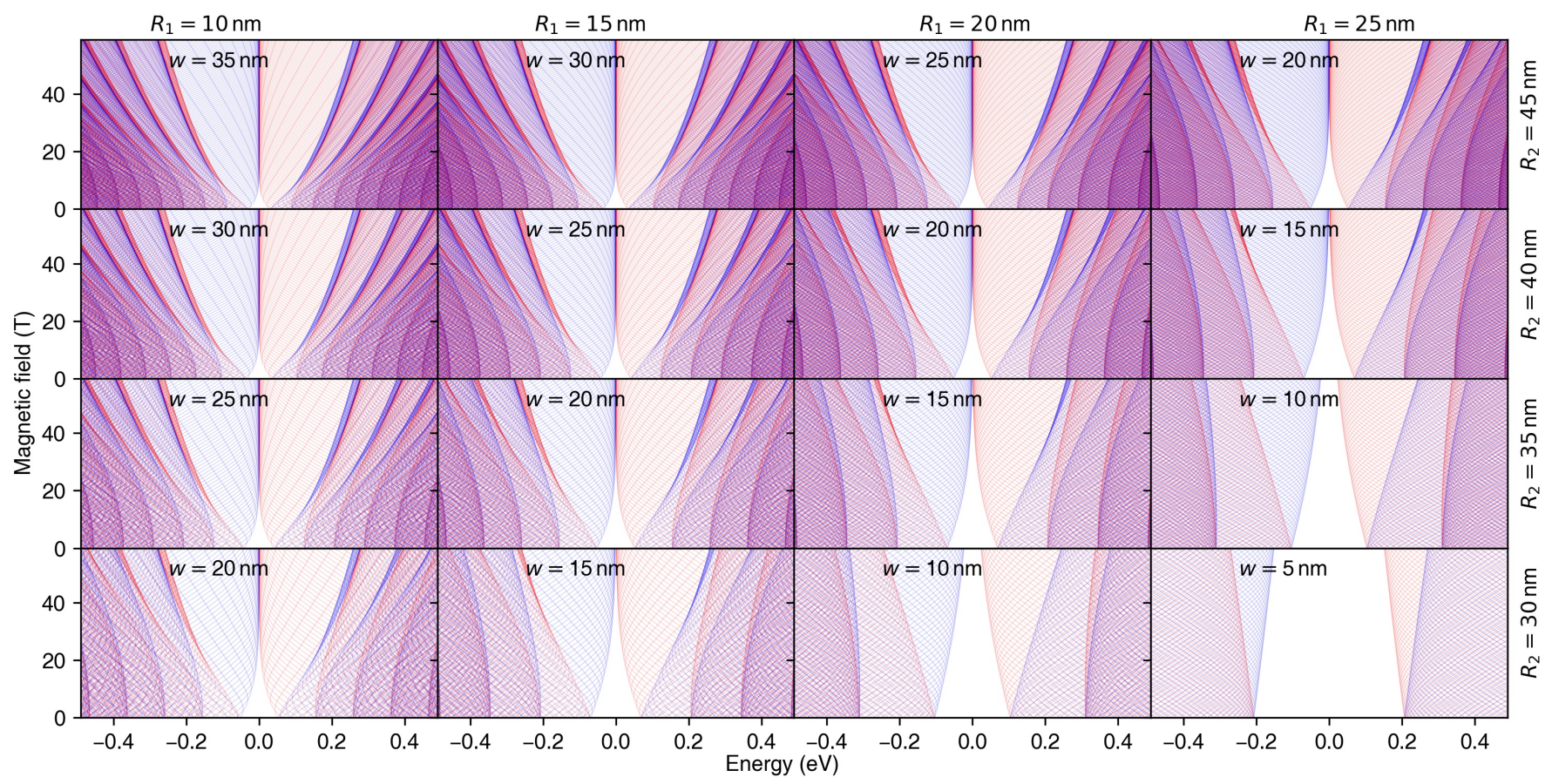

FIG. S6. Dirac ring eigenvalues: Eigenvalue spectrum for Dirac rings of various inner and outer radius, $R_{1}$ and $R_{2}$, respectively, as a function of magnetic field and energy. Red (blue) energy levels correspond to the $K\left(K^{\prime}\right)$ valley. All structures with the same width, $R_{2}-R_{1}$, have the same band gap and emergent band spacing.

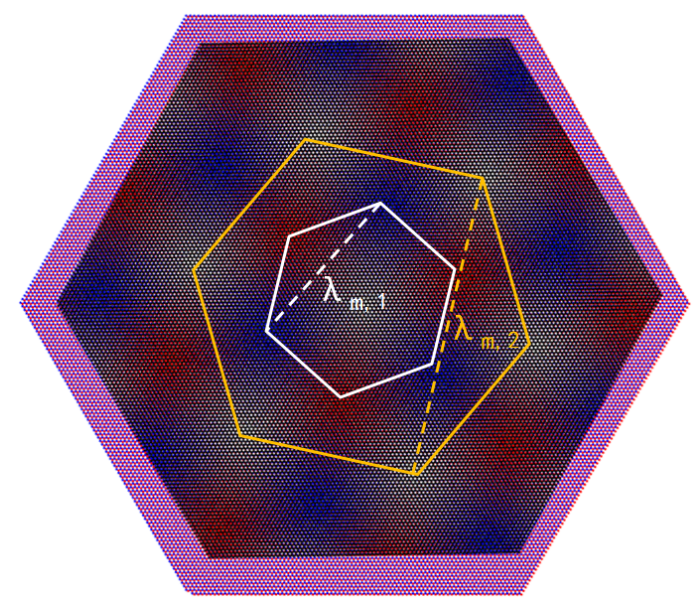

FIG. S7. Moiré lattice from graphene on hexagonal boron nitride: Schematic of the moiré pattern in graphene $/ \mathrm{hBN}$ heterostructure with a twist angle of $0.9^{\circ}$. Gray, blue and red dots indicate the location of the carbon, boron and nitrogen atoms. The unit cell and period, $\lambda_{m, 1}$, of the primary moiré superlattice is marked with white, and the larger unit cell and period, $\lambda_{m, 2}=\sqrt{3} \cdot \lambda_{m, 1}$, of the secondary moiré is marked with an orange hexagon [S12].

we attribute the larger activation energy to the gap induced by nanostructuring of the graphene. However, the larger gap was only evident in the Arrhenius plots at temperatures above $\sim 200 \mathrm{~K}$. Since our measurement setup does not support heating, we were unable to extract more data points, and the exact value extracted here should thus be used with some caution. It does, however, fit well with the expected value of the half-gap, both from theory and from the zero-field half-gap estimation.

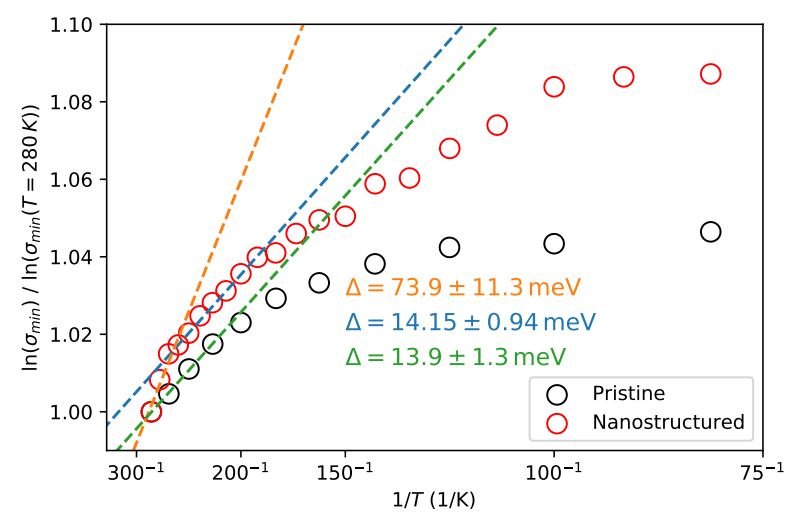

FIG. S8. Extraction of half-gaps from Arrhenius plots: Arrhenius plot of the temperature, $T$, vs minimum conductance, $\sigma_{\min }$, for the pristine and nanostructured graphene. At lower temperatures a small half-gap associated with the hBN-G moiré-interaction can be seen in both areas, with $\Delta \approx 14 \mathrm{meV}$, while at higher temperatures a larger half-gap of $\Delta \approx 74 \mathrm{meV}$ can be seen in the nanostructured device, which is associated with the half-gap due to lithographic nanostructuring. 


\subsection{Zero-field half-gap estimation of the nanostructured region}

The nanostructured region is insulating in a regime of $\Delta V_{G} \approx 2.1 \mathrm{~V}$, where the resistivity is higher than $h / e^{2}$. That is, the total conductance is smaller than the quantum conductance, even considering the four-fold carrier degeneracy of graphene, which is the same as the Ioffe-Regel criterion for localization in 2D semiconductors [S16, S17]. This width can be used to estimate the size of the half-gap,

$$
\Delta_{g a p}=E_{g} / 2=\hbar v_{F} \sqrt{\pi \alpha \Delta V_{G} / 2},
$$

similar to the approach used for other semiconducting materials based on graphene [S18, S19], yielding $\Delta_{\text {gap }}=$ $92 \mathrm{meV}$. Accounting for the $14 \mathrm{meV}$ moiré-induced halfgap, we thus estimate the size of the zero-field half-gap in the nanostructured region to be $\Delta_{0}=78 \mathrm{meV}$, corresponding to a width, $w=1 \mathrm{~nm} \cdot \mathrm{eV} / \Delta_{0}$, of $\sim 13 \mathrm{~nm}$, which matches very well the expected range of dimensions, $12-15 \mathrm{~nm}$, of the holes in the nanostructured area of the graphene device.

\subsection{Additional magnetotransport data of the pristine and nanopatterned areas}

In Fig. S9 we show additional magnetotransport data, which includes $\rho_{x x}, \rho_{x y}, \sigma_{x x}$, and $\sigma_{x y}$ of both the pristine and nanopatterned areas. The conductivity is calculated as $\sigma_{x x, x y}=\rho_{x x, x y} /\left(\rho_{x x}^{2}+\rho_{x y}^{2}\right)$, with $\rho_{x x}=(W / L) R_{x x}$ and $\rho_{x y}=R_{x y}$, where $L$ and $W$ are the length and width of the Hall-bar, respectively. For the data from the nanostructured area presented here, a small measurement artifact can be seen around $V_{G}=-1 \mathrm{~V}$, which did not show up at subsequent measurement cycles. For clarity, the artefact has been removed in the data presented in the main text.

We would like to highlight the $\rho_{x x}$ and $\rho_{x y}$ data from the nanostructured area in particular. From the $\rho_{x x}$ data, it is now much more clear how the insulating region, attributed to the formation of a band gap, decreases as the magnetic field strength is increased. The colorscale has been chosen such that the $\rho_{x x}=\mathrm{h} / \mathrm{e}^{2}$ is white, which helps highlight the transition from insulator to metal. For the $\rho_{x y}$ data, we draw attention to the relatively large signal at $B=0 \mathrm{~T}$, approximately for the same range of gate voltages as the width of the insulating region seen in the $\rho_{x x}$ data. This effect will be discussed below in the context of a band-gap induced non-zero Berry curvature.

\subsection{Investigation of non-zero $\rho_{x y}$ at $B=0 \mathrm{~T}$}

\subsubsection{Transport data}

The presence of a magnetic field gives rise to the Lorentz force, $\mathbf{F} \propto \mathbf{v} \times \mathbf{B}$, acting on charge carriers, which in turn gives rise to transverse currents; the Hall effect. Similarly, the presence of a non-zero Berry curvature, $\Omega$, gives rise to a force acting on momentum, $\mathbf{F} \propto \mathbf{p} \times \boldsymbol{\Omega}$, which is transverse to the applied electric field, i.e. the transport direction [S20]. In the gap of gapped graphene, this Berry curvature is given by

$$
\boldsymbol{\Omega}= \pm \frac{\hbar^{2} v_{F}^{2} \Delta}{2 \epsilon_{\mathbf{p}}^{3}}
$$

with the typical dispersion for gapped graphene given by

$$
\epsilon_{\mathbf{p}}=\sqrt{v_{F}^{2} \mathbf{p}^{2}+\Delta^{2}} .
$$

In other words, a finite zero-field $\rho_{x y}$ can be expected in single-layer graphene with a gap in the band structure. Such effects have indeed been observed in single-layer graphene with a moiré-induced band gap [S21] using non-local measurements to detect the topological currents, with $\rho_{x y}$ reaching values of $\sim 600 \Omega$.

In Fig. S10 we show zero-field $(B=0 \mathrm{~T})$ data for both the pristine and nanostructured areas of our device. For the pristine area with a moiré-induced half-gap, $\Delta_{m}=14 \mathrm{meV}$, we detect a small non-zero $\rho_{x y}$ reaching values of $\sim 0.006 \cdot h / e^{2}=150 \Omega$. For the nanostructured graphene, however, where we estimate a half-gap on the order of $\Delta_{0}=78 \mathrm{meV}$, we observe a relatively large $\rho_{x y}$ reaching values of $\sim 0.3 \cdot h / e^{2}=7700 \Omega$. This peak in $\rho_{x y}$ is centred around the insulating regime where $\rho_{x x}>h / e^{2}$, as highlighted by the shaded area, which is also expected from a sample with non-zero Berry curvature [S21].

However, as non-local measurements were not performed in this study, we will in the following section try to highlight other possible sources of this large zero-field transverse signal.

\subsubsection{COMSOL study}

A much simpler explanation of the finite $\rho_{x y}$ would be trivial geometric effects due to redirected current flow in the highly patterned region. To investigate if this can account for the experimental observations, we simulate the device with a full-scale finite element simulation (FES). The geometry was defined in COMSOL 5.3 as shown in Fig. S11a-b which closely matches the device in layout and proportions. The model was constructued by introducing two different materials, corresponding to the pristine region $(\mathrm{P})$, and a narrow fringe-like annulus zone (A) surrounding each hole, see Fig. S11b. The key parameter is the (spatial) electrical sensitivity, which is a well-established quantity that describes the influence of a local disturbance on a device-level electrical measurement variable, in this case the Hall resistance, as in Ref. [S22], where finite element sensitivity calculations such as 

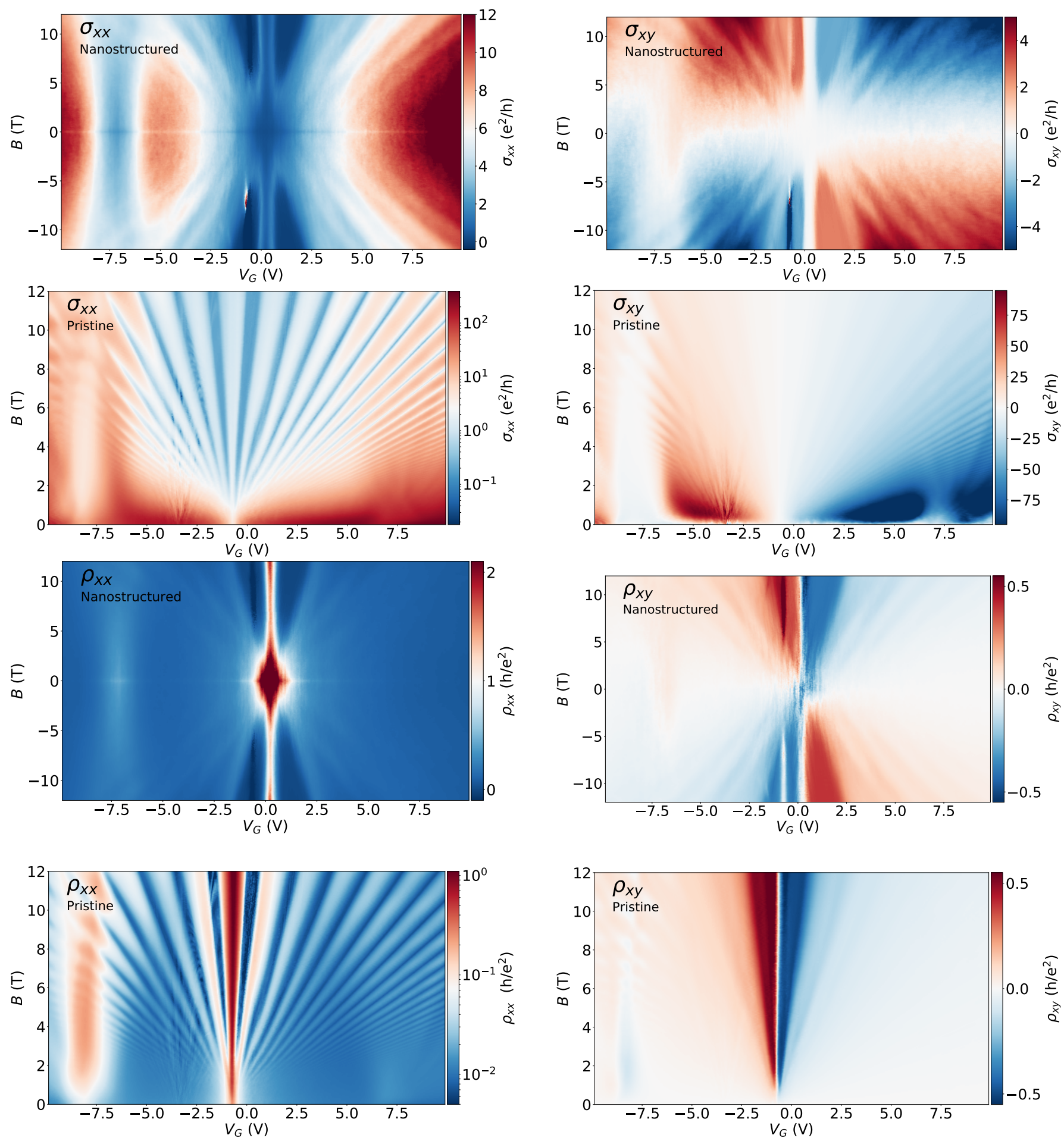

FIG. S9. Additional magnetotransport data: Additional magnetotransport data, $\rho_{x x}, \rho_{x y}, \sigma_{x x}$, and $\sigma_{x y}$, for the pristine and nanopatterned areas, as a function of applied magnetic field, $B$, and gate voltage, $V_{G}$. Base temperature was $\sim 4 \mathrm{~K}$.

ours are compared to magnetic scanning gate microscopy measurements.

Electrical sensitivity maps were generated with COMSOL 5.3, in the two relevant measurement configurations:
Hall configuration $\left(\mathrm{V}_{1}, \mathrm{~V}_{2}, \mathrm{~V}_{3}, \mathrm{~V}_{4}\right)=\left(\mathrm{S}, \mathrm{V}^{-}, \mathrm{V}^{+}, \mathrm{D}\right)$, using contact numbering from Fig. S11c. with source (S), drain (D) and measurement probes $\left(\mathrm{V}^{-}, \mathrm{V}^{+}\right)$, and HallPrime configuration, $\left(\mathrm{V}_{1}, \mathrm{~V}_{2}, \mathrm{~V}_{3}, \mathrm{~V}_{4}\right)=\left(\mathrm{V}^{-}, \mathrm{S}, \mathrm{D}, \mathrm{V}^{+}\right)$. 

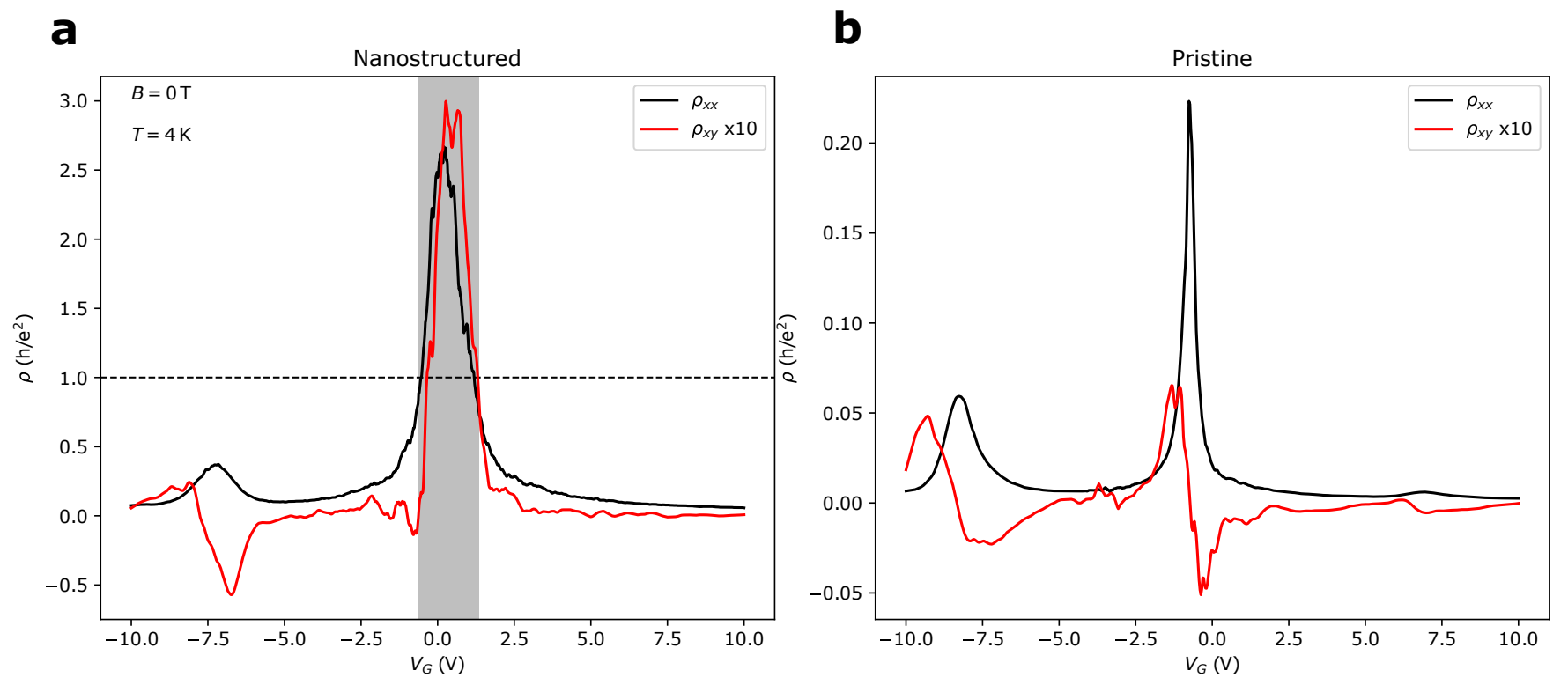

FIG. S10. Non-zero $\rho_{x y}$ at $\boldsymbol{B}=\mathbf{0} \mathbf{T}$ : Zero-field $(B=0 \mathrm{~T})$ longitudinal, $\rho_{x x}$, and transverse, $\rho_{x y}$, resistivity for the nanostructured (a) and pristine (b) graphene as a function of gate voltage, $V_{G}$. A large transverse signal is present in the nanostructured graphene, reaching $\rho_{x y}=0.3 \cdot h / e^{2}=7700 \Omega$.

The $x$ and $y$ component of the current density $\left(J_{x}, J_{y}\right)$ are simulated for both configurations. The sensitivity, $S$, for the Hall measurement was then calculated using [S23]

$$
S=J_{x} \cdot J_{x}^{\text {Prime }}+J_{y} \cdot J_{y}^{\text {Prime }} .
$$

The normalized sensitivity $\left(\iint S \mathrm{~d} x \mathrm{~d} y=1\right)$ is shown in Fig. S11c. The center of the Hall cross has zero sensitivity while areas of maximum sensitivity are observed at the corners of the cross. As a result, any geometrical asymmetries in those areas, for instance due to differences in the precise location of holes near the four corners, will potentially lead to a finite transverse signal at zero magnetic field. To investigate the response of $\rho_{x y}$ at $B=0 T$, gate dependent FES were performed using COMSOL 5.3. Both materials from Fig. S11a had a gate-dependent conductivity defined by [S24]

$$
\begin{gathered}
\sigma=n_{\text {tot }} \mu q, \\
n_{\text {tot }}=\sqrt{n_{0}^{2}+n^{2}}, \\
n=\frac{C_{\text {gate }}\left(V_{G}-V_{0}\right)}{q},
\end{gathered}
$$

with $\sigma$ being the conductivity, $n_{\text {tot }}$ the carrier concentration, $\mu$ the mobility, $q$ the electronic charge, $n_{0}$ the carrier concentration (due to charged impurities) at the charge neutrality point, $n$ the carriers induced by gate voltage, $V_{G}, C_{\text {gate }}$ is the capacitance per unit area of the gate, and $V_{0}$ is the gate voltage at the charge neutrality point. For both zones $\mathrm{P}$ and $\mathrm{A}$ in the model,
$V_{0}=0 \mathrm{~V}$ and $n=2.8 \cdot 10^{11} \mathrm{~cm}^{-2}$, with the mobilities being set to $15,000 \mathrm{~cm}^{2} \mathrm{~V}^{-1} \mathrm{~s}^{-1}$ and $600 \mathrm{~cm}^{2} \mathrm{~V}^{-1} \mathrm{~s}^{-1}$ for pristine and nanostructured area, respectively. A boundary current source was set at $10 \mu \mathrm{A}$, while the voltage difference was extracted in the $x$ and $y$ direction of the nanostructured and the pristine section. A parametric sweep of $V_{G}$ was performed from $-5 \mathrm{~V}$ to $+5 \mathrm{~V}$ with a stepsize of $20 \mathrm{mV}$. The results are shown in Fig. S11d-e. For $\rho_{x x}$ of the nanostructured graphene, the experimental and FES data are in good agreement. While $\rho_{x y} \sim 0$ in the pristine region as expected, a zero-field peak appears both in the FES and the experimental data for the nanostructured region. The important point to note here is that for the FES, the $\rho_{x y} / \rho_{x x}$ is a constant, i.e. the Hall signal is a small, constant fraction $(\sim 1 / 50)$ of the longitudinal conductivity. In contrast, the experimental data has a far more prominent $\rho_{x y}$ which does not scale with $\rho_{x x}$, as shown in Fig. S11f. We checked that the ratio $\rho_{x y} / \rho_{x x}$ does not flatten out upon addition of a random voltage offset, which could have occurred in the measurement. We also checked different placements of the superlattice on the Hall bar, which did not change the overall picture. The observation of a pronounced, finite $\rho_{x y}$ for the nanostructured region, which cannot be accounted for by trivial geometrical effects (asymmetric placement of holes in regions with high electrical sensitivity) supports the hypothesis that the Hall response testify the presence of a Berry curvature, as expected for gapped graphene. 

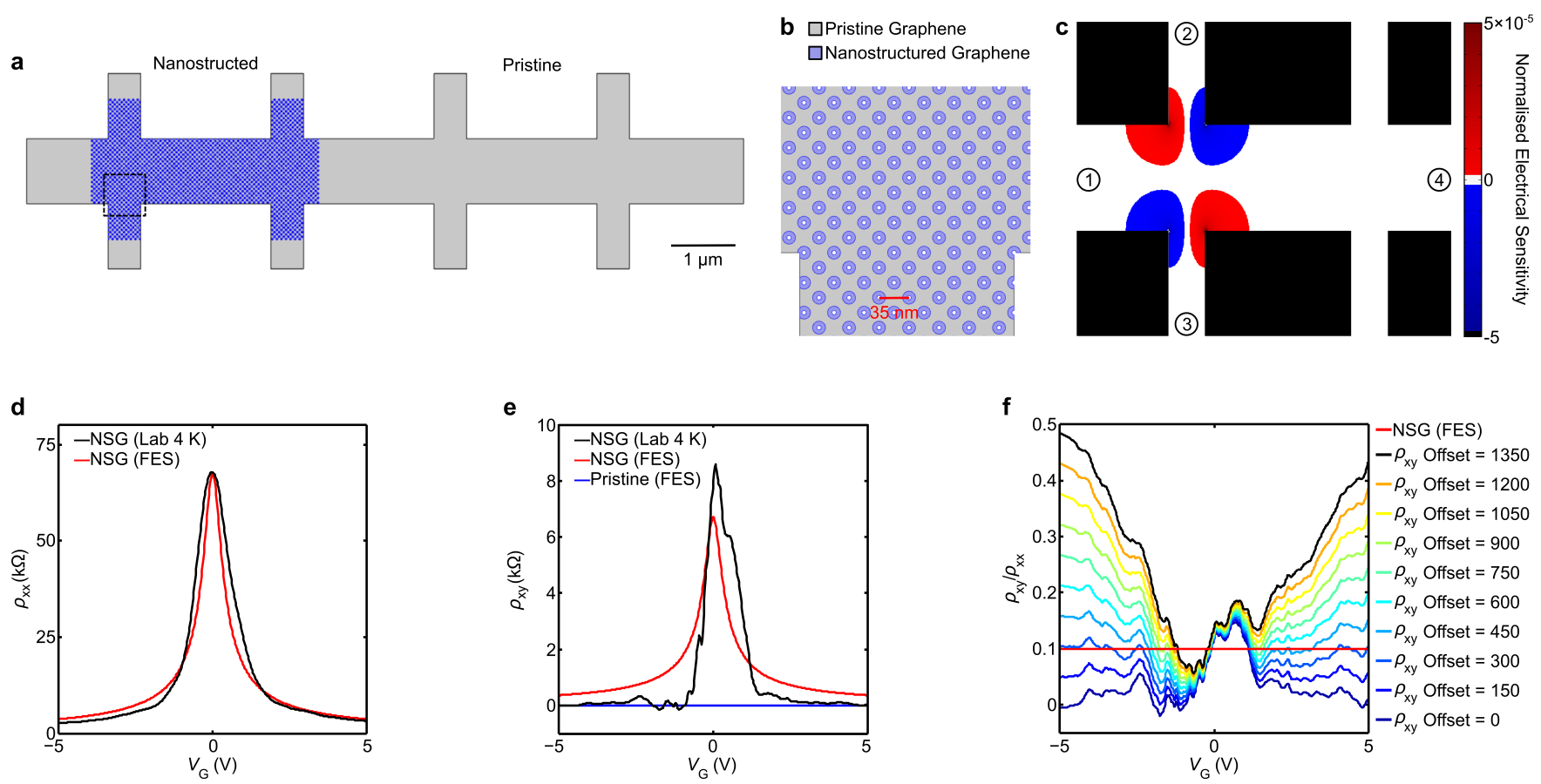

FIG. S11. COMSOL simulation details: (a) Geometry of the device discussed in the main text used for FES. (b) Zoomed image of the nanostructured area. (c) Normalised electrical sensitivity map for a Hall measurement with the geometery from a). (d) Comparison of the electrically measured $\rho_{x x}$ (black) and finite element simulation data for the nanostructured area (red). (e) Comparison of the electrically measured $\rho_{x y}$ (black) and finite element simulation data for the nanostructured area (red) and the pristine section (blue). (f) Ratio of $\rho_{x y} / \rho_{x x}$ for the nanostructured area for finite element simulation data (red) and electrically measured with various offsets added to the $\rho_{x y}$ data.

[S1] Pizzocchero, F. et al. The hot pick-up technique for batch assembly of van der waals heterostructures. Nat. Commun. 7, 11894 (2016).

[S2] Wang, L. et al. One-dimensional electrical contact to a two-dimensional material. Science 342, 614-617 (2013).

[S3] Oberhuber, F. et al. Weak localization and raman study of anisotropically etched graphene antidots. Applied Physics Letters 103, 143111 (2013).

[S4] Thomsen, M. R., Power, S. R., Jauho, A.-P. \& Pedersen, T. G. Magnetic edge states and magnetotransport in graphene antidot barriers. Phys. Rev. B 94, 045438 (2016).

[S5] Thomsen, M. R. \& Pedersen, T. G. Analytical dirac model of graphene rings, dots, and antidots in magnetic fields. Phys. Rev. B 95, 235427 (2017).

[S6] Johansson, F. et al. mpmath: a Python library for arbitrary-precision floating-point arithmetic (version 1.00) (2017). http://mpmath.org/.

[S7] Han, M. Y., Özyilmaz, B., Zhang, Y. \& Kim, P. Energy band-gap engineering of graphene nanoribbons. Phys. Rev. Lett. 98, 206805 (2007).

[S8] Pedersen, J. G. \& Pedersen, T. G. Hofstadter butterflies and magnetically induced band-gap quenching in graphene antidot lattices. Phys. Rev. B 87, 235404 (2013).

[S9] Pedersen, T. G., Jauho, A. P. \& Pedersen, K. Optical response and excitons in gapped graphene. Phys. Rev.
$B$ 79, 113406 (2009).

[S10] Yankowitz, M. et al. Emergence of superlattice dirac points in graphene on hexagonal boron nitride. Nat. Phys. 8, 382 (2012).

[S11] Dean, C. et al. Hofstadter's butterfly and the fractal quantum hall effect in moiré superlattices. Nature 497, 598 (2013).

[S12] Chen, G. et al. Emergence of tertiary dirac points in graphene moiré superlattices. Nano Lett. 17, 3576-3581 (2017).

[S13] Wang, L. et al. New generation of moir \'e superlattices in doubly aligned hbn/graphene/hbn heterostructures. arXiv preprint arXiv:1812.10031 (2018).

[S14] Taychatanapat, T. \& Jarillo-Herrero, P. Electronic transport in dual-gated bilayer graphene at large displacement fields. Physical review letters 105, 166601 (2010).

[S15] Hunt, B. et al. Massive dirac fermions and hofstadter butterfly in a van der waals heterostructure. Science 340, 1427-1430 (2013).

[S16] Sarma, S. D. \& Hwang, E. Two-dimensional metalinsulator transition as a strong localization induced crossover phenomenon. Phys. Rev. B 89, 235423 (2014).

[S17] Radisavljevic, B. \& Kis, A. Mobility engineering and a metal-insulator transition in monolayer $\operatorname{mos}_{2}$. Nat. Mater. 12, 815 (2013).

[S18] Han, M. Y., Brant, J. C. \& Kim, P. Electron transport 
in disordered graphene nanoribbons. Physical review letters 104, 056801 (2010).

[S19] Shimizu, T. et al. Large intrinsic energy bandgaps in annealed nanotube-derived graphene nanoribbons. $\mathrm{Na}$ ture nanotechnology 6, 45 (2011).

[S20] Xiao, D., Chang, M.-C. \& Niu, Q. Berry phase effects on electronic properties. Rev. Mod. Phys. 82, 1959 (2010).

[S21] Gorbachev, R. et al. Detecting topological currents in graphene superlattices. Science 346, 448-451 (2014).

[S22] Rajkumar, R. et al. Magnetic scanning gate microscopy of graphene hall devices. Journal of Applied Physics 115, 172606 (2014).

[S23] Koon, D. W., Wang, F., Petersen, D. H. \& Hansen, O. Sensitivity of resistive and hall measurements to local inhomogeneities: Finite-field, intensity, and area corrections. J. Appl. Phys. 116, 133706 (2014).

[S24] Mackenzie, D. M. et al. Quality assessment of graphene: Continuity, uniformity, and accuracy of mobility measurements. Nano Research 10, 3596-3605 (2017). 